\title{
Multi-mode piezoelectric shunt damping with residual mode correction by evaluation of modal charge and voltage
}

Toftekær, Johan Frederik; Høgsberg, Jan

Published in:

Journal of Intelligent Material Systems and Structures

Link to article, DOI:

$10.1177 / 1045389 \times 19891646$

Publication date:

2020

Document Version

Peer reviewed version

Link back to DTU Orbit

Citation (APA):

Toftekær, J. F., \& Høgsberg, J. (2020). Multi-mode piezoelectric shunt damping with residual mode correction by evaluation of modal charge and voltage. Journal of Intelligent Material Systems and Structures, 31(4), 570-586 . https://doi.org/10.1177/1045389x19891646

\section{General rights}

Copyright and moral rights for the publications made accessible in the public portal are retained by the authors and/or other copyright owners and it is a condition of accessing publications that users recognise and abide by the legal requirements associated with these rights.

- Users may download and print one copy of any publication from the public portal for the purpose of private study or research.

- You may not further distribute the material or use it for any profit-making activity or commercial gain

- You may freely distribute the URL identifying the publication in the public portal 


\title{
MULTI-MODE PIEZOELECTRIC SHUNT DAMPING WITH RESIDUAL MODE CORRECTION BY EVALUATION OF MODAL CHARGE AND VOLTAGE
}

\author{
JOHAN FREDERIK TOFTEKÆR AND JAN HØGSBERG \\ DEPARTMENT OF MECHANICAL ENGINEERING \\ TECHNICAL UNIVERSITY OF DENMARK \\ NILS KOPPELS ALLÉ, BUILDING 404, DK-2800 KGS. LYNGBY, DENMARK
}

\begin{abstract}
A resonant piezoelectric shunt tuning procedure is based on the limiting eigenvalue problems associated with short and open circuiting (SC and $\mathrm{OC}$ ) of the piezoelectric domains attached to a vibrating structure. Whereas SC and OC frequencies are directly obtained from the eigenvalues, the associated electrical equation further determines a modal charge as an SC reaction force and a modal voltage from the electric deflection in the OC limit. By a modal representation with SC mode shapes the structural equation fully decomposes, while the inherent contributions from non-resonant vibration modes in the corresponding electrical equation consistently define an effective modal capacitance, estimated by the modal charge to voltage ratio. The shunt tuning is obtained from the governing characteristic equation for the targeted vibration mode, in which the residual mode correction is explicitly represented by the effective modal capacitance. The tuning procedure with an effective modal capacitance is generalized to multiple piezoelectric domains with independent shunts for simultaneous damping of multiple vibration modes. The accuracy of the proposed shunt tuning methods is demonstrated by a numerical beam example with three piezoceramic patch pairs and independent resistive-inductive shunts. The analysis is carried out in a commercial finite element program, in which the required modal frequencies, charge and voltage are readily available as output to the SC and OC eigenvalue problems.
\end{abstract}

\section{INTRODUCTION}

Piezoelectric materials are characterized by their ability to couple mechanical strains and stresses with electrical fields and displacements. This property may be exploited for vibration mitigation of structures, by attaching piezoelectric transducers to the structure, whereby a fraction of the vibrational energy can be converted into electrical energy by the piezoelectric material and dissipated in an electric circuit or shunt wired between the two transducer electrodes.

A piezoelectric shunt may be composed in various designs by either passive [10, 11, 28, 29], semi-active $[20,5,2,3]$ or active $[1,23]$ electric circuits. The entirely passive piezoelectric shunt is attractive because of its independence of external power and unconditional stability. The simplest passive $(R)$ shunt is pure resistive, whereby the shunted piezoelectric material has viscoelastic properties [10]. The resonant shunt is obtained by adding an inductance $(L)$, designed to resonate with the inherent piezoelectric capacitance, as initially suggested and experimentally demonstrated by [8]. A tuning procedure based on a single-mode approximation of the electromechanical structure has been derived for the series $L R$-shunt by [10], with tuning formulas that explicitly depend on the inherent piezoelectric capacitance. The optimal tuning for the corresponding parallel shunt is subsequently proposed by [28], while the series and parallel shunts have been compared in [22] and [4]. Alternative methods for the optimum series and parallel shunt tuning has been suggested in $[25,30,12,24]$, all relying on modal truncation, whereby the inherent piezoelectric capacitance appears as a direct tuning parameter.

The main issue with the practical implementation of passive resonant shunt has traditionally been to obtain the high inductance values required for the damping of low-frequency vibrations.

Key words and phrases. Piezoelectric shunt damping, resonant shunt calibration, residual mode correction, effective electromechanical coupling coefficient. 
For experimental verification of shunt tuning methods, a so-called synthetic inductor therefore commonly emulates high inductance values [7]. A benefit of the synthetic inductor is that the emulated inductance is adjustable, whereby an exact prior inductance tuning is of less important. A major drawback of the synthetic inductor is the use of operational amplifiers, requiring constant power sources. Because resonant shunts are often realized by such semi-active circuits with operational amplifiers, further active or semi-active improvements of the shunts has naturally been suggested, for instance by the design of a negative capacitance $[20,2,3]$ or the use of switch damping [5]. However, recently high inductance values have been obtained passively by winding a cobber wire around a closed magnetic coil [18], permitting the construction of pure passive resonant shunts and electrical circuit analogies [19]. Contrary to a synthetic inductor, this passive component requires a precise prior tuning to determine the exact number of wire winds, whereby it may be necessary to include the effect of non-resonant vibration modes to obtain a sufficiently accurate shunt tuning.

Recently, the inaccuracy associated with the traditional use of the inherent piezoelectric capacitance as a direct tuning parameter has been recognized. In [2] the inherent capacitance of the piezoelectric material has thus been modified by the influence from higher vibration modes, while a theoretical study of the influence from an increasing number of vibration modes on the shunt tuning was provided in [9] for a simply supported plate. A Common denominator for these approaches is the inclusion and evaluation of additional resonant frequencies and mode shapes. In [15] this is avoided through the derivation of explicit expressions for the supplemental flexibility and inertia effects from the non-resonant vibration modes, thus explicitly correcting the tuning of tuned mass- and inerter-based absorbers. The equivalence to resonant piezoelectric shunt damping has subsequently been considered in [13], with [26] proposing an expression for the frequency dependent effective capacitance, which incorporates a consistent residual mode correction. As full system mass and stiffness matrices are not necessarily available in commercial finite element (FE) software, an alternative shunt tuning method in [27] relies on the so-called effective electromechanical coupling coefficient (EMCC) [14] at resonance, limited by the short and open circuit (SC and OC) conditions for the piezoelectric material. The proposed tuning formulas have thus been based on the effective EMCC and a modal charge in the SC limit, which are readily accessible quantities in any FE software supporting electromechanical coupled analysis. However, for shunt tuning based on experimental measurements the method proposed in [27] is less evident, since the modal charge assumes a particular normalization and the effective EMCC may be difficult to evaluate to a sufficient accuracy. Instead, the present paper provides a deeper insight to the influence of the non-resonant vibration modes, demonstrating that the optimum shunt tuning with residual mode correction may be determined from a modal charge and a modal voltage, obtained from the eivenvalue problems in the SC and OC conditions, respectively. Hereby the shunt tuning becomes independent of modal normalization and therefore suitable for both numerical and experimental implementation. Furthermore, the method is extended to multi-mode damping through the individual tuning of several shunted piezoelectric domains (absorbers).

The paper is structured in four main sections. In the section Discrete vibration problem the general electromechanical discrete vibration problem is presented, for which the SC and OC limits are considered. The OC resonant frequencies are inherently larger than the corresponding SC frequencies, due to the stiffening by the capacitance of the piezoelectric material. The traditional modal assumption states this stiffening effect to be inversely proportional to the inherent piezoelectric capacitance, while the exact stiffening, including non-resonant modal interaction, may be represented by an effective modal capacitance, evaluated directly from the stored modal charge in the SC condition and the modal voltage in the OC condition. Hereby, the governing characteristic equation for a representative 2-dof modal system of the full electromechanical structure is obtained and used in the section Single-mode calibration to determine the optimum single-mode calibration of multiple piezoelectric absorbers. In the section Multi mode calibration the effect of having piezoelectric absorbers tuned to simultaneously suppress several resonant vibrations is investigated and two methods for the optimum shunt calibration are proposed. The former considers the effect of the piezoelectric absorbers tuned to other vibration modes to be insignificant, whereby the optimum tuning to any resonant vibration mode may be evaluated from the same SC 


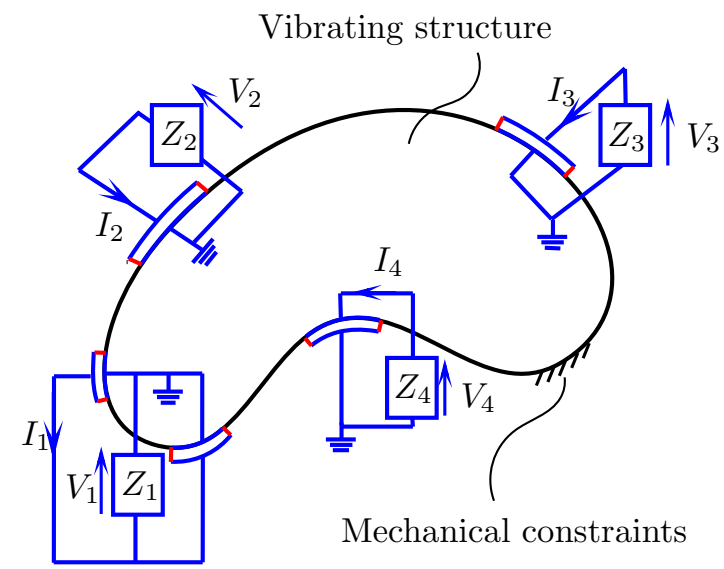

FigURE 1. Vibrating structure with attached piezoelectric domains comprised by $N_{p}=4$ piezoelectric absorbers with independent shunt impedance $Z_{i}$.

and OC eigenvalue problems and a modified effective EMCC. An enhanced method also includes the influence from the piezoelectric absorbers tuned to other vibration modes by considering them either in their SC or OC condition. Finally, in the section Numerical example the single-mode and two multi-mode shunt tuning methods are analyzed and compared by a numerical example concerning a simply supported beam with three pairs of shunted piezoceramic patches.

\section{Discrete Vibration PRoblem}

The coupled vibration problem of a vibrating structure with attached piezoelectric domains and $i=1,2, \ldots, N_{p}$ independent shunts $Z_{i}$, see Figure 1 , may be discretized by finite elements $(\mathrm{FE})$ and written according to $[27]$ as

$$
\left(\left[\begin{array}{cc}
K^{E} & k_{m e}^{E} \\
\left(k_{m e}^{E}\right)^{t} & -C_{p}
\end{array}\right]-\omega^{2}\left[\begin{array}{cc}
M & 0 \\
0 & 0
\end{array}\right]\right)\left\{\begin{array}{l}
U \\
V
\end{array}\right\}=\left\{\begin{array}{c}
0 \\
-Q
\end{array}\right\}
$$

Here $\left[K^{E}\right]$ is the elastic stiffness matrix for the structure with SC piezoelectric electrodes $\left(Z_{i}=0\right)$, while $[M]$ is the mass matrix. The coupling between the mechanical and the electrical domains is governed by the coupling matrix $\left[k_{m e}^{E}\right]$ with rows equal to the number of mechanical degrees of freedom (dofs) and columns equal to the number of independent piezoelectric shunts $N_{p}$, while $(\ldots)^{t}$ represents the transpose operation.

A piezoelectric shunt may be wired to several piezoelectric domains and act as a single resulting piezoelectric absorber, as long as two interface electrodes can be defined, where one remains grounded at all times, see Figure 1. The electrical domain is described by a diagonal matrix $\left[C_{p}\right]$ containing the resulting modified blocked capacitance $\left(\bar{C}_{p}^{\epsilon^{S}}\right)_{i}$ for each piezoelectric absorber [27]. The total number of dofs in the discrete system is given by the mechanical displacements in $\{U\}$ and the voltages in $\{V\}$ between each pair of grounded and non-grounded electrodes. Finally, the vector $\{Q\}$ contains the stored electric charge on each of the non-grounded electrodes, while the corresponding time-derivatives provide the flow of current represented in the frequency domain as

$$
I_{i}=\mathrm{i} \omega Q_{i}
$$

and shown in Figure 1 between each pair of grounded and non-grounded electrodes, with $i=\sqrt{-1}$ denoting the imaginary unit.

2.1. Electromechanical coupling. The influence of the piezoelectric absorbers on the vibrating structure is characterized by two limiting eigenvalue problems associated with $\mathrm{SC}$ and $\mathrm{OC}$ piezoelectric electrodes. 
The SC condition is defined by zero voltage between the piezoelectric electrodes $\left(Z_{i}=0\right)$, for which the current is free to flow. The corresponding eigenvalue problem thus follows from $\{V\}=\{0\}$ in (1),

$$
\left(\left[K^{E}\right]-\omega_{j}^{2}[M]\right)\left\{U_{j}\right\}=\{0\}
$$

where $\omega_{j}$ is the SC frequency and $\left\{U_{j}\right\}$ the corresponding SC mode shape vector of vibration mode $j$. The second equation in (1) provides a stored modal charge on each non-grounded electrode as

$$
\left\{Q_{j}\right\}=-\left[k_{m e}^{E}\right]^{t}\left\{U_{j}\right\}
$$

Assuming harmonic oscillations with SC frequency $\omega_{j}$, the magnitude of the stored modal charge may alternatively be determined by the corresponding flow of current $\left\{Q_{j}\right\}=\left\{I_{j}\right\} /\left(\mathrm{i} \omega_{j}\right)$ from (2).

In the opposite OC condition, no current flows between the piezoelectric electrodes $\left(Z_{i} \rightarrow \infty\right.$ in Figure 1), equivalent to vanishing charge. The OC eigenvalue problem thus follows from (1) with $\{Q\}=\{0\}$, conveniently formulated as

$$
\left(\left[K^{E}\right]+\left[k_{m e}^{E}\right]\left[C_{p}\right]^{-1}\left[k_{m e}^{E}\right]^{t}-\hat{\omega}_{j}^{2}[M]\right)\left\{\hat{U}_{j}\right\}=\{0\}
$$

where $\{V\}$ has been eliminated by the second equation in (1). In (5), $\hat{\omega}_{j}$ and $\left\{\hat{U}_{j}\right\}$ are respectively the OC frequency and mode shape of vibration mode $j$. The second equation of the discrete system in (1) determines the corresponding modal electric response, which in this case is given by a modal voltage between each pair of grounded and non-grounded electrodes,

$$
\left\{\hat{V}_{j}\right\}=\left[C_{p}\right]^{-1}\left[k_{m e}^{E}\right]^{t}\left\{\hat{U}_{j}\right\}
$$

In the remainder of the present paper, a similar normalization of the SC $\left\{U_{j}\right\}$ and $\mathrm{OC}\left\{\hat{U}_{j}\right\}$ mode shape vectors is assumed, for instance by normalizing the mode shapes to unit modal mass.

The rate of convertible energy by the piezoelectric domains may now be determined for a resonant mode $j$ by the effective EMCC, defined as the relative difference between the squared $\mathrm{SC}$ and $\mathrm{OC}$ frequencies,

$$
\kappa_{j}^{2}=\frac{\hat{\omega}_{j}^{2}-\omega_{j}^{2}}{\omega_{j}^{2}}
$$

It is noted that $\hat{\omega}_{j}^{2} \geq \omega_{j}^{2}$ due to the quadratic projection of the coupling matrix $\left[k_{m e}^{E}\right]$ on the capacitance matrix $\left[C_{p}\right]$ in (5). Thus, the effective EMCC is also a measure of the relative stiffness increase by the OC piezoelectric domains on the vibrating structure. The effective EMCC may alternatively be determined by considering the OC eigenvalue problem (5) multiplied with the SC mode shape $\left\{U_{j}\right\}^{t}$,

$$
\begin{array}{r}
\left\{U_{j}\right\}^{t}\left[K^{E}\right]\left\{\hat{U}_{j}\right\}+\left\{U_{j}\right\}^{t}\left[k_{m e}^{E}\right]\left[C_{p}\right]^{-1}\left[k_{m e}^{E}\right]^{t}\left\{\hat{U}_{j}\right\} \\
-\hat{\omega}_{j}^{2}\left\{U_{j}\right\}^{t}[M]\left\{\hat{U}_{j}\right\}=\{0\}
\end{array}
$$

After division with an intermediate modal mass,

$$
\tilde{m}_{j}=\left\{U_{j}\right\}^{t}[M]\left\{\hat{U}_{j}\right\}
$$

and introduction of the SC modal charge (4) and the OC modal voltage (6), the equation (8) can be written as

$$
\omega_{j}^{2}-\frac{\left\{Q_{j}\right\}^{t}\left\{\hat{V}_{j}\right\}}{\tilde{m}_{j}}-\hat{\omega}_{j}^{2}=0
$$

Considering the relation between the SC and $\mathrm{OC}$ resonant frequencies defined by (7), it is found from (10) that the effective EMCC can alternatively be determined as

$$
\kappa_{j}^{2}=-\frac{\left\{Q_{j}\right\}^{t}\left\{\hat{V}_{j}\right\}}{\tilde{m}_{j} \omega_{j}^{2}}
$$

This expression for the effective EMCC is conveniently used in the following, where a modal decomposition of the general vibration problem (1) is considered. It is noted that the combined modal projection used here is proposed as basis for a modal reduction technique in [17], which is found to improve the response accuracy compared to traditional modal reduction methods. This result is in a agreement with the findings in the subsequent section Modal equations, in which 
the influence from the non-resonant vibration modes is included through the combined modal projection.

2.2. Modal equations. The general vibration problem (1) may be decoupled by introducing a modal representation with the SC mode shapes of the mechanical vibrations $\{U\}=[U]\{v\}$, where the matrix $[U]$ contains $\left\{U_{j}\right\}$ as columns and $\{v\}$ represents the $N$ modal coordinates $v_{j}$. Introducing this modal representation into (1) and multiplying with the transpose of the SC mode shapes $[U]^{t}$, the vibration problem (1) can be written as

$$
\begin{aligned}
{[U]^{t}\left(\left[K^{E}\right]-\omega^{2}[M]\right)[U]\{v\}+[U]^{t}\left[k_{m e}^{E}\right]\{V\} } & =\{0\}, \\
{\left[k_{m e}^{E}\right]^{t}[U]\{v\}-\left[C_{p}\right]\{V\} } & =-\{Q\}
\end{aligned}
$$

The top set of mechanical equations in (12) uncouple and may for vibration mode $j$ be written as

$$
\left(\omega_{j}^{2}-\omega^{2}\right) v_{j}-\frac{\left\{Q_{j}\right\}^{t}}{m_{j}}\{V\}=0, \quad j=1,2, \ldots, N
$$

where $\left\{Q_{j}\right\}$ has been introduced as in (4). Each of the bottom $N_{p}$ electric equations in (12) still depends on the full range of modal coordinates $v_{j}$,

$$
\sum_{j=1}^{N}\left\{Q_{j}\right\} v_{j}=\{Q\}-\left[C_{p}\right]\{V\}
$$

It is inconvenient to evaluate the full range of modal coefficients and charge components. Thus, a modal approximation of the left hand-side in (14) is introduced. In order to maintain the accuracy of the model, the effect of the non-resonant modes is included and subsequently approximated around a target resonant mode $j=r$ by e.g. a flexibility and an inertia correction term according to [15]. In [26] these flexibility and inertia effects have been demonstrated to correspond to a capacitance and an inductance, respectively, altering the resulting modified blocked capacitance $\bar{C}_{p}^{\epsilon^{S}}$ at resonance. In the present analysis, this additional capacitance from the non-resonant vibration modes is determined by considering the $\mathrm{SC}$ and $\mathrm{OC}$ limits.

It follows from the modal equation (13) that $v_{j}$ is proportional to the voltage vector $\{V\}$, whereby the sum over the non-resonant vibration modes in (14) contributes with an additional term to the blocked capacitance,

$$
\begin{aligned}
\left\{Q_{r}\right\} v_{r} & =\{Q\}-\left(\left[C_{p}\right]+\sum_{j \neq r}^{N} \frac{\left\{Q_{j}\right\}\left\{Q_{j}\right\}^{t}}{m_{j} \omega_{j}^{2}} \frac{\omega_{j}^{2}}{\omega_{j}^{2}-\omega^{2}}\right)\{V\} \\
& =\{Q\}-\left[C_{r}\right]\{V\}
\end{aligned}
$$

in which the introduced matrix $\left[C_{r}\right]$ is assumed diagonal, deliberately ignoring any coupling effects from the summation inside the parenthesis. Hereby the diagonal of $\left[C_{r}\right]$ contains the effective modal capacitance around the target vibration mode $r$, for each piezoelectric absorber.

The modal coefficient for target mode $r$ may be determined from (15) by multiplying with the modal voltage $\left\{\hat{V}_{r}\right\}^{t}$ and then dividing with the scalar product $\left\{\hat{V}_{r}\right\}^{t}\left\{Q_{r}\right\}$,

$$
v_{r}=\frac{\left\{\hat{V}_{r}\right\}^{t}}{\left\{\hat{V}_{r}\right\}^{t}\left\{Q_{r}\right\}}\{Q\}-\frac{\left\{\hat{V}_{r}\right\}^{t}\left[C_{r}\right]}{\left\{\hat{V}_{r}\right\}^{t}\left\{Q_{r}\right\}}\{V\}
$$

This target modal coordinate $v_{r}$ may now be inserted into (13), whereby a governing modal equation is obtained as

$$
\begin{aligned}
\left(\omega_{r}^{2}-\omega^{2}\right)\left(\frac{\left\{\hat{V}_{r}\right\}^{t}\{Q\}}{\left\{\hat{V}_{r}\right\}^{t}\left\{Q_{r}\right\}}-\frac{\left\{\hat{V}_{r}\right\}^{t}\left[C_{r}\right]\{V\}}{\left\{\hat{V}_{r}\right\}^{t}\left\{Q_{r}\right\}}\right) & \\
-\frac{\left\{Q_{r}\right\}^{t}\{V\}}{m_{r}} & =0
\end{aligned}
$$

The impedance relation for each shunt is then introduced as

$$
\{V\}=-\mathrm{i} \omega[Z(\omega)]\{Q\}
$$


where $[Z(\omega)]$ is a diagonal matrix containing the impedance $Z_{i}(\omega)$ for shunt $i$ in the diagonal. The impedance relation (18) is used in (17) to eliminate $\{Q\}$, whereby it can be written in homogeneous form,

$$
\begin{aligned}
&\left(\omega_{r}^{2}-\omega^{2}\right)\left(\frac{\left\{\hat{V}_{r}\right\}^{t}[Z(\omega)]^{-1}\{V\}}{i \omega\left\{\hat{V}_{r}\right\}^{t}\left\{Q_{r}\right\}}+\frac{\left\{\hat{V}_{r}\right\}^{t}\left[C_{r}\right]\{V\}}{\left\{\hat{V}_{r}\right\}^{t}\left\{Q_{r}\right\}}\right) \\
&+\frac{\left\{Q_{r}\right\}^{t}\{V\}}{m_{r}}=0
\end{aligned}
$$

The expression inside the square brackets constitutes $N_{p}$ independent characteristic equations, since both $[Z(\omega)]$ and $\left[C_{r}\right]$ are diagonal matrices. The independent equation for a piezoelectric shunt $i$ may therefore be written as

$$
\begin{aligned}
&\left(\omega_{r}^{2}-\omega^{2}\right)\left(\frac{\left(\hat{V}_{r}\right)_{i}}{i \omega Z_{i}(\omega)\left\{\hat{V}_{r}\right\}^{t}\left\{Q_{r}\right\}}+\frac{\left(\hat{V}_{r}\right)_{i}\left(C_{r}\right)_{i}}{\left\{\hat{V}_{r}\right\}^{t}\left\{Q_{r}\right\}}\right) \\
&+\frac{\left(Q_{r}\right)_{i}}{m_{r}}=0
\end{aligned}
$$

The effective capacitances $\left(C_{r}\right)_{i}$ for the targeted resonant mode $r$ may now be determined from (20) in the OC limit $\left(Z_{i}(\omega) \rightarrow \infty\right)$, at which it reduces to

$$
\left(\omega_{r}^{2}-\hat{\omega}_{r}^{2}\right) \frac{\left(\hat{V}_{r}\right)_{i}\left(C_{r}\right)_{i}}{\left\{\hat{V}_{r}\right\}^{t}\left\{Q_{r}\right\}}+\frac{\left(Q_{r}\right)_{i}}{m_{r}}=0
$$

when $\omega=\hat{\omega}_{r}$ has been introduced. The relation between the SC and OC resonant frequencies may then be introduced by the previous expression for the effective EMCCs in (7) or (11). Hereby, shunt $i$ 's effective capacitance $\left(C_{r}\right)_{i}$ for the targeted mode $r$ is determined from (21) as

$$
\begin{aligned}
\left(C_{r}\right)_{i} & =\frac{\left\{\hat{V}_{r}\right\}^{t}\left\{Q_{r}\right\}}{m_{r} \omega_{r}^{2}} \frac{\omega_{r}^{2}}{\hat{\omega}_{r}^{2}-\omega_{r}^{2}} \frac{\left(Q_{r}\right)_{i}}{\left(\hat{V}_{r}\right)_{i}} \\
& =-\frac{\left(Q_{r}\right)_{i}}{\left(\hat{V}_{r}\right)_{i}} \frac{\tilde{m}_{r}}{m_{r}} \simeq-\frac{\left(Q_{r}\right)_{i}}{\left(\hat{V}_{r}\right)_{i}}
\end{aligned}
$$

where the last approximation $\tilde{m}_{r} \simeq m_{r}$ is valid for most practical problems with limited electromechanical coupling. Furthermore, it should be noted that the minus in the effective capacitance expression requires that the sign convention of the SC and OC mode shapes is the same, whereby $\left(Q_{r}\right)_{i}$ and $\left(\hat{V}_{r}\right)_{i}$ always have opposite signs. This may be circumvented by simply introducing absolute values of both $\left|Q_{r}\right|_{i}$ and $\left|\hat{V}_{r}\right|_{i}$, as applied later.

The effective capacitance, comprising both the resulting blocked capacitance and any contributions from non-resonant vibration modes, is found to be accurately approximated by the absolute value of the SC modal charge $\left|Q_{r}\right|_{i}$ divided by the OC modal voltage $\left|\hat{V}_{r}\right|_{i}$. The effective capacitance (22) also implies an alternative definition of the effective EMCC obtained by elimination of $\left\{\hat{V}_{j}\right\}$ in $(11)$,

$$
\kappa_{r}^{2}=\frac{\left\{Q_{r}\right\}^{t}\left[C_{r}\right]^{-1}\left\{Q_{r}\right\}}{m_{r} \omega_{r}^{2}}
$$

This expression for the effective EMCC is similar to the often used modal EMCC [21] without nonresonant mode contributions, where the resulting blocked capacitance $\left[C_{p}\right]$ is here simply replaced by the effective capacitance $\left[C_{r}\right]$.

Finally, the characteristic equation for target mode $r$, represented by the square brackets in (19), may be re-written by multiplication with $\left[C_{r}\right]^{-1}\left\{Q_{r}\right\}$ and division by $\omega_{r}^{2}$,

$$
\begin{aligned}
&\left(1-\frac{\omega^{2}}{\omega_{r}^{2}}\right)\left(\frac{\omega^{2}}{\omega_{r}^{2}}-\mathrm{i} \frac{\omega}{\omega_{r}} \frac{\left\{\hat{V}_{r}\right\}^{t}[Z(\omega)]^{-1}\left[C_{r}\right]^{-1}\left\{Q_{r}\right\}}{\left\{\hat{V}_{r}\right\}^{t}\left\{Q_{r}\right\} \omega_{r}}\right) \\
&+\kappa_{r}^{2} \frac{\omega^{2}}{\omega_{r}^{2}}=0
\end{aligned}
$$


In the following sections, this characteristic equation (24) is used to obtain optimum single- and multi-mode shunt calibration by inserting the respective shunt impedances and compare the corresponding characteristic equations with the generic equation from [16],

$$
\frac{\omega^{4}}{\omega_{r}^{4}}-\left(2+4 \chi^{2}\right) \frac{\omega^{2}}{\omega_{r}^{2}}+1+4 i \lambda \chi \frac{\omega}{\omega_{r}}\left(1-\frac{\omega^{2}}{\omega_{r}^{2}}\right)=0
$$

This generic equation ensures equal modal damping through the tuning of the shunt inductance $L$, while the subsequent tuning of the shunt resistance $R$ by $\lambda=1 / \sqrt{2}$ balances large attainable damping and effective vibration amplitude mitigation. It finally follows from [16] that the damping ratio for the target mode $r$ can be estimated as

$$
\zeta_{r} \simeq \lambda \chi=\sqrt{\frac{1}{2} \chi^{2}}
$$

where $\chi^{2}$ is proportional to the effective EMCC [12], as also demonstrated in the following.

\section{Single-MOde CALibration}

This section presents the tuning of $N_{p}$ independent piezoelectric absorbers to a single target vibration mode $r$. The corresponding characteristic equation is given by (24), in which the term depending on the impedance and capacitance may be replaced by a sum over the $N_{p}$ absorbers tuned to mode $r$. When inserting the effective capacitances (22), the characteristic equation (24) can be written as

$$
\begin{aligned}
&\left(1-\frac{\omega^{2}}{\omega_{r}^{2}}\right)\left(\frac{\omega^{2}}{\omega_{r}^{2}}+\mathrm{i} \omega \sum_{i=1}^{N_{p}} \frac{\left(\hat{V}_{r}\right)_{i}\left(Q_{r}\right)_{i}}{\left\{\hat{V}_{r}\right\}^{t}\left\{Q_{r}\right\}} \frac{\left(\hat{V}_{r}\right)_{i}}{Z_{i}(\omega)\left(Q_{r}\right)_{i} \omega_{r}^{2}}\right) \\
&+\kappa_{r}^{2} \frac{\omega^{2}}{\omega_{r}^{2}}=0
\end{aligned}
$$

The optimum tuning for the parallel and series shunts is then derived by introducing the corresponding impedances into (27) followed by comparison with the generic equation (25) to obtain the balanced calibration principle for resonant absorbers [16].

3.1. Parallel shunt tuning. The parallel shunt impedance $Z_{i}=Z_{i}^{p}$ is conveniently expressed in terms of the inverse shunt inductance and resistance for shunt $i$ as (superscript $p$ refers to parallel)

$$
\frac{1}{Z_{i}^{p}(\omega)}=\frac{1}{R_{i}^{p}}+\frac{1}{\mathrm{i} \omega L_{i}^{p}}
$$

When inserting this parallel shunt impedance, the characteristic equation (27) can be written in accordance with the generic form (25) as

$$
\begin{gathered}
\frac{\omega^{4}}{\omega_{r}^{4}}-\left(1+\kappa_{r}^{2}-\sum_{i=1}^{N_{p}} \frac{\left(\hat{V}_{r}\right)_{i}\left(Q_{r}\right)_{i}}{\left\{\hat{V}_{r}\right\}^{t}\left\{Q_{r}\right\}} \frac{1}{L_{i}^{p}} \frac{\left(\hat{V}_{r}\right)_{i}}{\left(Q_{r}\right)_{i} \omega_{r}^{2}}\right) \frac{\omega^{2}}{\omega_{r}^{2}} \\
-\sum_{i=1}^{N_{p}} \frac{\left(\hat{V}_{r}\right)_{i}\left(Q_{r}\right)_{i}}{\left\{\hat{V}_{r}\right\}^{t}\left\{Q_{r}\right\}} \frac{1}{L_{i}^{p}} \frac{\left(\hat{V}_{r}\right)_{i}}{\left(Q_{r}\right)_{i} \omega_{r}^{2}} \\
-\mathrm{i} \frac{\omega}{\omega_{r}} \sum_{i=1}^{N_{p}} \frac{\left(\hat{V}_{r}\right)_{i}\left(Q_{r}\right)_{i}}{\left\{\hat{V}_{r}\right\}^{t}\left\{Q_{r}\right\}} \frac{1}{R_{i}^{p}} \frac{\left(\hat{V}_{r}\right)_{i}}{\left(Q_{r}\right)_{i} \omega_{r}}\left(1-\frac{\omega^{2}}{\omega_{r}^{2}}\right)=0
\end{gathered}
$$

Comparison with (25) requires a unit constant term in (29). As the first fraction inside the summation of the constant term in (29) sums to unity, the inductances

$$
L_{i}^{p}=-\frac{\left(\hat{V}_{r}\right)_{i}}{\left(Q_{r}\right)_{i} \omega_{r}^{2}}
$$

secure the desired unit constant term. It is seen that (30) corresponds to the traditional modal inductance tuning $L_{i}^{p}\left(C_{r}\right)_{i} \omega_{r}^{2}=1$ with the effective capacitance $\left(C_{r}\right)_{i}$ replacing the inherent 
TABLE 1. Optimum parallel and series single-mode, modified single-mode and multi-mode calibration for shunt $i$ tuned with respect to target mode $r$ and the corresponding effective EMCCs and damping ratios.

\begin{tabular}{|c|c|c|c|c|c|c|}
\hline \multirow{2}{*}{ 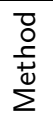 } & \multicolumn{2}{|c|}{ Inductance } & \multicolumn{2}{|c|}{ Resistance } & \multirow{2}{*}{$\begin{array}{c}\text { EMCC } \\
\kappa_{r}^{2}\end{array}$} & \multirow{2}{*}{$\begin{array}{c}\text { Damping } \\
\quad \zeta_{r}\end{array}$} \\
\hline & $L_{i}^{p}$ & $L_{i}^{s}$ & $R_{i}^{p}$ & $R_{i}^{s}$ & & \\
\hline $\begin{array}{l}\frac{0}{60} \\
\stackrel{50}{5}\end{array}$ & $\frac{\left|\hat{V}_{r}\right|_{i}}{\left|Q_{r}\right|_{i} \omega_{r}^{2}}$ & $\frac{\left|\hat{V}_{r}\right|_{i}}{\left|Q_{r}\right|_{i} \hat{\omega}_{r}^{2}} \frac{\omega_{r}^{2}}{\hat{\omega}_{r}^{2}}$ & $\frac{\left|\hat{V}_{r}\right|_{i}}{\left|Q_{r}\right|_{i} \omega_{r}} \sqrt{\frac{1}{2 \kappa_{r}^{2}}}$ & $\frac{\left|\hat{V}_{r}\right|_{i}}{\left|Q_{r}\right|_{i} \hat{\omega}_{r}} \frac{\omega_{r}^{2}}{\hat{\omega}_{r}^{2}} \sqrt{2 \kappa_{r}^{2}}$ & $\frac{\hat{\omega}_{r}^{2}-\omega_{r}^{2}}{\omega_{r}^{2}}$ & $\overline{\frac{1}{8} \kappa_{r}^{2}}$ \\
\hline 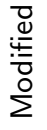 & $\frac{\left|\hat{V}_{r}\right|_{i}}{\left|Q_{r}\right|_{i} \omega_{r}^{2}}$ & $\frac{\left|\hat{V}_{r}\right|_{i}}{\left|Q_{r}\right|_{i} \hat{\omega}_{r}^{2}} \frac{\omega_{r}^{2}}{\hat{\omega}_{r}^{2}}$ & $\frac{\left|\hat{V}_{r}\right|_{i}}{\left|Q_{r}\right|_{i} \omega_{r}} \sqrt{\frac{1}{2 \widetilde{\kappa}_{r}^{2}}}$ & $\frac{\left|\hat{V}_{r}\right|_{i}}{\left|Q_{r}\right|_{i} \hat{\omega}_{r}} \frac{\omega_{r}^{2}}{\hat{\omega}_{r}^{2}} \sqrt{2 \tilde{\kappa}_{r}^{2}}$ & $\sum_{i=1}^{n_{p}} \frac{\left(Q_{r}\right)_{i}\left(\hat{V}_{r}\right)_{i}}{\left\{Q_{r}\right\}^{t}\left\{\hat{V}_{r}\right\}} \kappa_{r}^{2}$ & \\
\hline$\frac{\frac{0}{2}}{\frac{2}{\pi}}$ & $\frac{\left|\underline{\hat{V}}_{r}\right|_{i}}{\left|\underline{Q}_{r}\right|_{i} \underline{\omega}_{r}^{2}}$ & $\frac{\left|\underline{\hat{V}}_{r}\right|_{i}}{\left|\underline{Q}_{r}\right|_{i} \underline{\hat{\omega}}_{r}^{2}} \frac{\underline{\omega}_{r}^{2}}{\underline{\hat{\omega}}_{r}^{2}}$ & $\frac{\left|\underline{\hat{V}}_{r}\right|_{i}}{\left|\underline{Q}_{r}\right|_{i} \underline{\omega}_{r}} \sqrt{\frac{1}{2 \underline{\kappa}_{r}^{2}}}$ & $\frac{\left|\underline{\hat{V}}_{r}\right|_{i}}{\left|\underline{Q}_{r}\right|_{i} \underline{\hat{\omega}}_{r}} \underline{\underline{\hat{\omega}}}_{r}^{2} \sqrt{2 \underline{\kappa}_{r}^{2}}$ & $\frac{\underline{\hat{\omega}}_{r}^{2}-\underline{\omega}_{r}^{2}}{\underline{\omega}_{r}^{2}}$ & $\sqrt{\frac{1}{8} \underline{\kappa}_{r}^{2}}$ \\
\hline
\end{tabular}

blocked capacitance $C_{p}^{\epsilon^{S}}$. When all $N_{p}$ inductances are optimally tuned, the parameter $\chi$ can be identified as $\chi^{2}=\kappa_{r}^{2} / 4$ from comparison of the quadratic terms, whereafter the shunt resistances are determined by comparing the common factor to the odd-power terms in (25) and (29),

$$
R_{i}^{p}=-\frac{\left(\hat{V}_{r}\right)_{i}}{\left(Q_{r}\right)_{i} \omega_{r}} \sqrt{\frac{1}{2 \kappa_{r}^{2}}}
$$

It is noted that the minus signs in the tuning formulas (30) and (31) appear because of the implied opposite signs between $\left(Q_{r}\right)_{i}$ and $\left(\hat{V}_{r}\right)_{i}$, as discussed previously. This may be circumvented by simply using the corresponding absolute values, whereby the tuning formulas can be written as presented in the first row of Table 1.

3.2. Series shunt tuning. The series impedance $Z_{i}=Z_{i}^{s}$ for shunt $i$ is given as (superscript $s$ refers to series)

$$
Z_{i}^{s}(\omega)=R_{i}^{s}+\mathrm{i} \omega L_{i}^{s}
$$

The impedances in the characteristic equation (27) are replaced by the series impedances (32) and the characteristic equation may then be written in accordance with the generic form (25),

$$
\begin{aligned}
& \frac{\omega^{4}}{\hat{\omega}_{r}^{4}}-(1\left.-\sum_{i=1}^{N_{p}} \frac{\left\{\hat{V}_{r}\right\}^{t}\left\{Q_{r}\right\}}{\left(\hat{V}_{r}\right)_{i}\left(Q_{r}\right)_{i}} \frac{1}{L_{i}^{s}} \frac{\left(\hat{V}_{r}\right)_{i}}{\left(Q_{r}\right)_{i} \hat{\omega}_{r}^{2}}\right) \frac{\omega^{2}}{\hat{\omega}_{r}^{2}} \\
&- \sum_{i=1}^{N_{p}} \frac{\left\{\hat{V}_{r}\right\}^{t}\left\{Q_{r}\right\}}{\left(\hat{V}_{r}\right)_{i}\left(Q_{r}\right)_{i}} \frac{1}{L_{i}^{s}} \frac{\left(\hat{V}_{r}\right)_{i}}{\left(Q_{r}\right)_{i} \hat{\omega}_{r}^{2}\left(1+\kappa_{r}^{2}\right)} \\
&+\mathrm{i} \frac{\omega}{\hat{\omega}_{r}} \frac{\sum_{i=1}^{N_{p}}\left(Q_{r}\right)_{i}^{2} R_{i}^{s}}{\sum_{i=1}^{N_{p}}\left(Q_{r}\right)_{i}^{2} L_{i}^{s} \hat{\omega}_{r}}\left(1-\frac{\omega^{2}}{\hat{\omega}_{r}^{2}}\right)=0
\end{aligned}
$$

expressed relative to the OC resonance frequency $\hat{\omega}_{r}$. For a detailed derivation of (33), see Appendix I. As for the parallel shunt the characteristic equation (33) is now compared with the generic equation (25), whereby the optimum series inductance is determined as

$$
L_{i}^{s}=-\frac{\left(\hat{V}_{r}\right)_{i}}{\left(Q_{r}\right)_{i} \hat{\omega}_{r}^{2}\left(1+\kappa_{r}^{2}\right)}=-\frac{\left(\hat{V}_{r}\right)_{i}}{\left(Q_{r}\right)_{i} \hat{\omega}_{r}^{2}} \frac{\omega_{r}^{2}}{\hat{\omega}_{r}^{2}}
$$

ensuring that the constant term in (33) becomes unity.

The parameter $\chi^{2}=\kappa_{r}^{2} / 4$ is the same as for the parallel shunt. The optimum tuning of the series resistance follows by inserting the series inductances (34) into (33) and then comparing the 


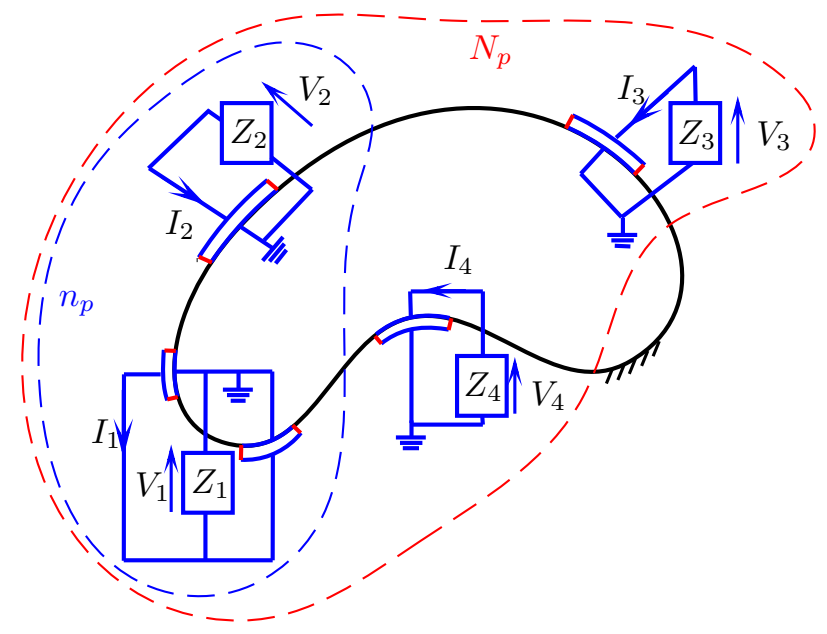

FiguRE 2. Vibrating structure with attached piezoelectric domains and $N_{p}=4$ independent shunts (red dashed). The subset $n_{p}$ (blue dashed) indicates the piezoelectric absorbers tuned to a target mode $r$.

odd-term factors in (25) and (33), which gives

$$
R_{i}^{s}=-\frac{\left(\hat{V}_{r}\right)_{i}}{\left(Q_{r}\right)_{i} \hat{\omega}_{r}\left(1+\kappa_{r}^{2}\right)} \sqrt{2 \kappa_{r}^{2}}=-\frac{\left(\hat{V}_{r}\right)_{i}}{\left(Q_{r}\right)_{i} \hat{\omega}_{r}} \frac{\omega_{r}^{2}}{\hat{\omega}_{r}^{2}} \sqrt{2 \kappa_{r}^{2}}
$$

The optimum series shunt tuning is also summarized in the first row of Table 1 , which also defines the effective EMCC and the attainable damping ratio $\zeta_{r}$ from (26).

\section{Multi mode CALiBration}

This section presents simultaneous damping of multiple vibration modes. Each piezoelectric absorber is still considered to be optimally tuned to a single target vibration mode. This implies that a reduced number of absorbers $n_{p}$ is tuned to a specific target mode $r$, whereby $n_{p}$ becomes a subset of the total number of absorbers $N_{p}$, as indicated by the blue dashed group in Figure 2 .

4.1. Modified single-mode calibration. When $n_{p} \subset N_{p}$ the single-mode calibration presented in the section Single-mode calibration will not provide the optimum resonant tuning with respect to mode $r$, mainly because the effective EMCC in (7) includes all $N_{p}$ piezoelectric absorbers. The tuning of the shunt inductances (30) and (34) are both independent of the effective EMCC. Hence, the inaccuracy of the single-mode calibration used on a subset of the piezoelectric absorbers $n_{p}$ mainly concerns the tuning of the shunt resistances in (31) and (35), which cause an apparent decrease in the attainable damping $\zeta_{r}$. By comparing the common factor to the odd-power terms in the characteristic equations (29) and (33) with the generic equation (25) it can be found that the damping ratio $\zeta_{r}$ from (26) is reduced by $\sum_{i=1}^{n_{p}}\left(\hat{V}_{r}\right)_{i}\left(Q_{r}\right)_{i}$ for the $n_{p}$ absorbers relative to the total amount $\left\{\hat{V}_{r}\right\}^{t}\left\{Q_{r}\right\}$ for all $N_{p}$ absorbers,

$$
\zeta_{r}=\sum_{i=1}^{n_{p}} \frac{\left(\hat{V}_{r}\right)_{i}\left(Q_{r}\right)_{i}}{\left\{\hat{V}_{r}\right\}^{t}\left\{Q_{r}\right\}} \sqrt{\frac{1}{8} \kappa_{r}^{2}}
$$

This reduction in attainable damping may be circumvented without additional computational costs by instead evaluating a modified effective EMCC, which only includes the subset of piezoelectric absorbers $n_{p}$ tuned to the target mode $r$. This modified effective EMCC $\tilde{\kappa}_{r}^{2}$ follows from (11) as

$$
\tilde{\kappa}_{r}^{2}=-\sum_{i=1}^{n_{p}} \frac{\left(Q_{r}\right)_{i}\left(\hat{V}_{r}\right)_{i}}{\tilde{m}_{r} \omega_{r}^{2}}=\sum_{i=1}^{n_{p}} \frac{\left(Q_{r}\right)_{i}\left(\hat{V}_{r}\right)_{i}}{\left\{Q_{r}\right\}^{t}\left\{\hat{V}_{r}\right\}} \kappa_{r}^{2}
$$


which is the original effective EMCC scaled by the same factor that in (36) reduces the damping ratio. The tilde $(\ldots)$ will in the following refer to this modified single-mode calibration of the reduced $n_{p}$ shunts. From this modified effective EMCC an enhanced resistance tuning is then simply obtained by replacing the effective EMCC $\kappa_{r}^{2}$ in (31) and (35) with its modified counterpart $\tilde{\kappa}_{r}^{2}$. The modified single-mode tuning formulas are provided in the second row of Table 1 and used for multi-mode damping in the numerical example in the section Numerical example.

The single-mode and modified single-mode calibrations are now compared in a root-locus diagram obtained by evaluating the full eigenvalue problem (1), with $\{Q\}$ eliminated by the parallel shunt impedances in (28), for the simply supported beam considered in the numerical example of the section Numerical example. The eigenvalue problems are established and evaluated using the in-house FE-code developed and described in [26]. In Figure 3 the roots around the first three resonant frequencies of the simply supported beam are plotted for varying resistance values, when each shunt circuit is tuned to a single target mode, as outlined in the section Triple mode damping. The single-mode and modified single-mode methods (black and red dots) are seen to follow the same root trajectories, which almost describe a semi-circle in the complex plane, indicating that the common inductance calibrations are close to optimum with equal modal damping. However, the optimum resistance tuning is seen to be off for the single-mode calibration (black crosses), as one of the two complex roots approaches the real axis along the right trajectory in Figure 3 . The modified resistance tuning (red crosses) is however seen to be in better agreement with the balanced calibration principle [16], with roots approximately placed on $\pm 45^{\circ}$ lines from the respective undamped SC frequencies.

Although the enhanced resistance tuning in many cases will provide an almost optimum resonant shunt tuning and a flat plateau in the frequency response curve around the target resonant frequency, it is not the exact optimum tuning, since the modal charge and voltage and the corresponding SC and $\mathrm{OC}$ frequencies are influenced by the effect of the piezoelectric absorbers not tuned with respect to target mode $r$, whereby the trajectories (with black and red dots) in Figure 3 do not meet at a bifurcation point. This modal interaction is neglected when simply using the modified effective EMCC in (37). A more precise shunt tuning may thus be obtained by reevaluating the SC and OC eigenvalue problems, in which the piezoelectric absorbers not tuned to mode $r$ are included either in their SC or OC state, depending on whether they are targeted to a mode $j$ above or below $r$.

4.2. Multi-mode calibration with non-resonant absorbers. In the modified single-mode calibration the influence from the piezoelectric absorbers not tuned with respect to mode $r$ is assumed insignificant, whereby the governing SC and OC eigenvalue problems may be represented by (3) and (5) with all $N_{p}$ piezoelectric domains jointly in SC and OC conditions, respectively. However, the influence from the piezoelectric absorbers not tuned with respect to mode $r$ may instead be included by considering them in either SC or OC condition, depending on whether an individual shunt targets a mode below or above the present tuning mode $r$.

By inserting the optimum inductance and resistance tuning from the first row of Table 1 into the respective parallel (28) and series (32) shunt impedances, these can be written for the resonant mode $r$ as

$$
\begin{aligned}
\frac{1}{Z_{i}^{p}(\omega)} & =\frac{\left|Q_{r}\right|_{i} \omega_{r}}{\left|\hat{V}_{r}\right|_{i}}\left(\sqrt{2 \kappa_{r}^{2}}-\mathrm{i} \frac{\omega_{r}}{\omega}\right), \\
Z_{i}^{s}(\omega) & =\frac{\left|\hat{V}_{r}\right|_{i}}{\left|Q_{r}\right|_{i} \hat{\omega}_{r}}\left(\sqrt{2 \kappa_{r}^{2}}+\mathrm{i} \frac{\omega}{\hat{\omega}_{r}}\right) \frac{\omega_{r}}{\hat{\omega}_{r}}
\end{aligned}
$$

Considering the shunt tuning to the target mode $r$, it can be seen from (38) that the absolute value of the parallel and series impedances of the piezoelectric absorbers tuned to a mode $j \neq r$ can be described as

$$
\begin{aligned}
& \left|Z_{i}^{p}\left(\omega_{r}\right)\right|,\left|Z_{i}^{s}\left(\omega_{r}\right)\right| \rightarrow \infty \text { for } \omega_{j}<<\omega_{r} \\
& \left|Z_{i}^{p}\left(\omega_{r}\right)\right|,\left|Z_{i}^{s}\left(\omega_{r}\right)\right| \rightarrow 0 \text { for } \omega_{j}>>\omega_{r}
\end{aligned}
$$


(a)
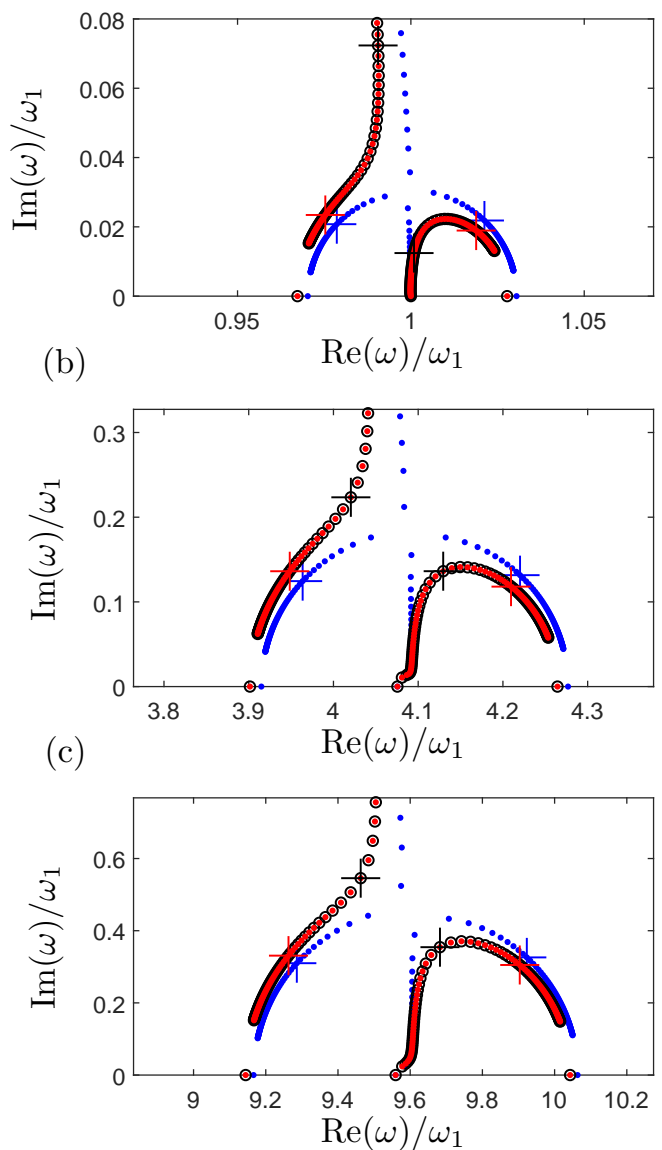

Figure 3. Root-locus diagram based on the numerical example in the section Numerical example and simultaneous three mode damping in Triple mode damping, plotted around mode 1 (a), 2 (b) and 3 (c) for the singlemode $(\circ)$, modified single-mode $(\bullet)$ and multi-mode $(\bullet)$ calibration and varying resistance values. The roots to the corresponding optimum resistance tuning are indicated by $(+),(+)$ and $(+)$.

An approximation of the influence from the piezoelectric absorbers not tuned with respect to mode $r$ is therefore obtained by keeping the piezoelectric absorbers tuned with respect to mode $j<r$ in the OC condition, while the absorbers tuned with respect to mode $j>r$ are in the SC condition when evaluating the SC and OC eigenvalue problems for the present target mode $r$. The $\mathrm{SC}$ eigenvalue problem and corresponding modal charge may thus be determined as

$$
\begin{array}{r}
\left(\left[K^{E}\right]+\left[k_{m e}^{E}\right]_{j<r}\left[C_{p}\right]_{j<r}^{-1}\left[k_{m e}^{E}\right]_{j<r}^{t}-\underline{\omega}_{r}^{2}[M]\right)\left\{\underline{U}_{r}\right\}=\{0\}, \\
\left\{\underline{Q}_{r}\right\}=-\left[k_{m e}^{E}\right]_{r}^{t}\left\{\underline{U}_{r}\right\}
\end{array}
$$

in which the OC stiffness contribution from the piezoelectric absorbers tuned to mode(s) $j<r$ will increase the original SC frequency $\underline{\omega}_{r} \geq \omega_{r}$, while the modal charges are evaluated only for the piezoelectric absorbers tuned with respect to mode $r$. 
The OC eigenvalue problem and corresponding modal voltage are further given as

$$
\begin{array}{r}
\left(\left[K^{E}\right]+\left[k_{m e}^{E}\right]\left[C_{p}\right]^{-1}\left[k_{m e}^{E}\right]^{t}-\left[k_{m e}^{E}\right]_{j>r}\left[C_{p}\right]_{j>r}^{-1}\left[k_{m e}^{E}\right]_{j>r}^{t}\right. \\
\left.-\underline{\hat{\omega}}_{r}^{2}[M]\right)\left\{\underline{\hat{U}}_{r}\right\}=\{0\}, \\
\left\{\underline{\hat{V}}_{r}\right\}=\left[C_{p}\right]_{r}^{-1}\left[k_{m e}^{E}\right]_{r}^{t}\left\{\underline{\hat{U}}_{r}\right\}
\end{array}
$$

in which the OC stiffness contribution from the piezoelectric absorbers tuned to mode(s) $j>r$ is subtracted from the original OC stiffness matrix in (41), whereby the original OC frequency decreases $\underline{\hat{\omega}}_{r} \leq \hat{\omega}_{r}$. Furthermore, the modal voltages are now evaluated only for the piezoelectric absorbers tuned with respect to mode $r$.

After evaluation of the SC and OC eigenvalue problems (40) and (41), a new effective EMCC can further be determined for a target mode $r$ as

$$
\underline{\kappa}_{r}^{2}=\frac{\underline{\hat{\omega}}_{r}^{2}-\underline{\omega}_{r}^{2}}{\underline{\omega}_{r}^{2}}
$$

The underbar will in the following denote the modal parameters for these new SC and OC eigenvalue problems with more advanced book keeping, including the state of the non-resonant shunts.

The optimum parallel and series shunt tuning then follows by the same tuning formulas proposed for the single-mode calibration, but with frequencies, modal charges and modal voltages based on the SC and OC eigenvalue problems in (40) and (41). The tuning formulas are provided in the third row of Table 1 .

Using this shunt tuning, the root trajectories for varying resistance are seen to follow semi-circles around the targeted resonant frequencies (blue dots) in Figure 3, while the optimum resistance tuning (blue crosses) appear on the $\pm 45^{\circ}$ lines from the respective undamped SC frequencies, with a corresponding damping ratio that can be estimated accurately as

$$
\underline{\zeta}_{r}=\sqrt{\frac{1}{8} \underline{\kappa}_{r}^{2}}
$$

The present multi-mode calibration method requires the evaluation of two eigenvalue problems for each target mode $r$, while tuning to any resonant mode may be determined by evaluating only two eigenvalue problems when using the single-mode calibration in the section Single-mode calibration and the modified single-mode calibration in Modified single-mode calibration. In the numerical example of the section Numerical example it is thus investigated how well the previous singlemode and modified single-mode calibrations perform compared to the multi-mode calibration of this section for a simply supported beam with three pairs of shunted piezoceramic patches, simoultaneuously targeting modes 1 to 3 .

\section{Numerical EXAMPle}

In the present section the calibration methods presented in the section Single-mode calibration and 4 are demonstrated by the analysis of a simple numerical example. The example concerns a simply supported beam with three pairs of piezoceramic patches, which can be seen in Figure 4 . All the piezoceramic patches have the same polarisation and are connected in parallel in pairs with a resonant shunt circuit $Z_{i}^{p}$. The dimensions of the beam are $40 \times 3 \times 0.2 \mathrm{~cm}^{3}$, while the piezoceramic patch pairs are placed with their respective centers in the quarter, half and threequarter point locations along the beam. The dimensions of the piezoceramic patches are $5 \times 3 \times 0.1$ $\mathrm{cm}^{3}$. The material properties of the beam are defined for aluminum by a Young's modulus $E=70$ GPa, a density $\rho_{b}=2700 \mathrm{~kg} / \mathrm{m}^{3}$ and a Poisson's ratio $\nu=0.33$. PZT $5 \mathrm{H}$ is the material considered for the piezoceramic patches with material properties that can be accessed through the "eFunda portal" [6]. The beam and piezoceramic patches are discretized by respectively $48 \times 4 \times 1$ and $6 \times 4 \times 120$-node three dimensional solid elements in ANSYS ${ }^{\circledR}$. The equipotential condition of the piezocelectric electrodes are enforced by a coupling constraint on the concerned electrical dofs, reducing the number of electrical dofs for each electrode to a single dof defined in a so-called master node [27]. The master nodes of the top and bottom interface electrodes are coupled, while 


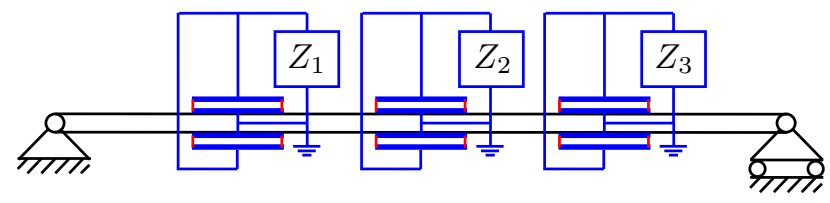

Figure 4. Simply supported beam with three pairs of shunted piezoceramic patches (absorbers).

TABLE 2. SC and OC frequencies and the effective EMCC of the simply supported beam with three piezoelectric absorbers.

\begin{tabular}{cccc}
\hline Mode & $\omega_{r} /(2 \pi)[\mathrm{Hz}]$ & $\hat{\omega}_{r} /(2 \pi)[\mathrm{Hz}]$ & $\kappa_{r}^{2}[\%]$ \\
\hline 1 & 25.18 & 25.35 & 1.41 \\
2 & 102.8 & 103.5 & 1.53 \\
3 & 242.0 & 244.2 & 1.79 \\
\hline
\end{tabular}

(a)

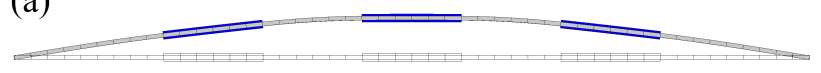

(b)

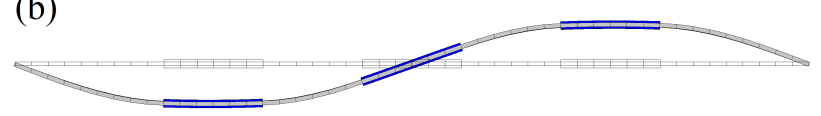

(c)

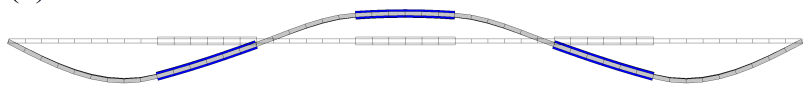

FiguRE 5. The first three mode shapes (a-c) of the simply supported beam with three pairs of SC piezocermic patches.

the inner interface electrodes are coupled and grounded, in order to model the parallel connection of each piezoceramic patch pair. In the following each of the three parallel connected piezoceramic patch pairs will therefore act as a single combined piezoelectric absorber with shunt impedance $Z_{i}^{p}(i=1,2$ or 3$)$.

Optimum piezoelectric shunt tuning is considered for the first three resonant vibration modes of the simply supported beam in Figure 4. Firstly, the three piezoelectric absorbers are in turn tuned with respect to a single target mode. Secondly, simultaneous damping of modes 1 and 2 is considered and finally simultaneous damping of modes 1, 2 and 3 is analyzed. Initially, the SC and OC eigenvalue problems are evaluated in order to determine the resonant frequencies, the effective EMCC and the corresponding mode shapes of the simply supported beam. The SC eigenvalue problem (3) is established by enforcing zero electric potential at all interface electrodes, while the OC eigenvalue problem (5) follows by deleting the zero potential constraints on the top/bottom interface electrodes of the three piezoelectric patch pairs. The first three SC and OC resonant frequencies and the corresponding effective EMCCs are provided in Table 2. The three mode shapes corresponding to the SC resonant frequencies in Table 2 can be seen in Figure 5, where the gray and blue colors indicate the beam and piezoelectric absorbers, respectively. It can be seen from Figure $5(\mathrm{a}, \mathrm{c})$ that the second piezoelectric absorber $\left(Z_{2}\right)$ is placed optimally for vibration modes 1 and 3 (the location of maximum curvature), while the position of the first $\left(Z_{1}\right)$ and third $\left(Z_{3}\right)$ piezoelectric absorbers are optimally located for mode 2 in Figure $5(\mathrm{~b})$. Moreover, it can be seen from Figure $5(\mathrm{~b})$ that the second piezoelectric absorber $\left(Z_{2}\right)$ is positioned at a nodal point for the second mode shape, which means that this absorber is without electromechanical coupling for vibration mode 2 , for which it can therefore not be applied. 
TABLE 3. Modal charge and voltage and optimum parallel and series single-mode calibration for each piezoelectric absorber to the first three resonant vibration modes.

\begin{tabular}{|c|c|c|c|c|c|c|c|}
\hline Shunt & $\left|Q_{r}\right|_{i}$ & {$\left[m\left(\frac{C s}{r a d}\right)^{2}\right.$} & $\left|\hat{V}_{r}\right|_{i}[\mathrm{k} V]$ & $L_{i}^{p}[\mathrm{H}]$ & $R_{i}^{p}[\mathrm{k} \Omega]$ & $L_{i}^{s}[\mathrm{H}]$ & $R_{i}^{s}[\mathrm{k} \Omega]$ \\
\hline$\neg Z_{1}$ & & 2.670 & 32.94 & 493.0 & 464.7 & 479.4 & 12.81 \\
\hline$\stackrel{ \pm}{\square} Z_{2}$ & & 3.781 & 46.64 & 493.0 & 464.7 & 479.4 & 12.81 \\
\hline$\sum^{\circ} Z_{3}$ & & 2.670 & 32.94 & 493.0 & 464.7 & 479.4 & 12.81 \\
\hline$\sim Z_{1}$ & & 16.06 & 198.4 & 29.62 & 109.3 & 28.73 & 3.269 \\
\hline$\frac{ \pm}{0} Z_{2}$ & & - & - & - & - & - & - \\
\hline$\sum^{\circ} Z_{3}$ & & 16.06 & 198.4 & 29.62 & 109.3 & 28.73 & 3.269 \\
\hline$m Z_{1}$ & & 28.86 & 356.0 & 5.335 & 42.91 & 5.150 & 1.494 \\
\hline$\stackrel{ \pm}{0} Z_{2}$ & & 41.05 & 506.5 & 5.335 & 42.91 & 5.150 & 1.494 \\
\hline$\sum^{\circ} Z_{3}$ & & 28.86 & 356.0 & 5.335 & 42.91 & 5.150 & 1.494 \\
\hline
\end{tabular}

5.1. Single mode damping. The optimum piezoelectric shunt tuning is now considered for the case where all three piezoelectric shunts are simultaneously tuned to the same target mode $r$. The single-mode calibration formulas, provided in the first row of Table 1, are thus used to determine the optimum parallel and series shunt tuning, given in Table 3. Furthermore, Table 3 also gives the modal charge (4) and voltage (6) found for each piezoelectric absorber from the ANSYS ${ }^{\circledR}$ model as an SC electric "reaction force" and an OC electric "displacement" in the master node of the top/bottom electrodes, respectively. It is noted that both the SC and OC mode shapes are scaled in order to obtain unit modal mass, which ensures that the (units) provided for modal charge and voltage are the mass normalized quantities.

The magnitude of the normalized modal charges and voltages in Table 3 determine the authority of the corresponding piezoelectric absorber on the targeted vibration mode. The largest modal charge and voltage is thus found for the second absorber $\left(Z_{2}\right)$ in mode 1 and 3 and for the first $\left(Z_{1}\right)$ and third $\left(Z_{3}\right)$ absorbers in mode 2 . As expected, zero electric charge and voltage is observed for the second absorber $\left(Z_{2}\right)$ in mode 2 . It can also be seen from Table 3 that the tuning of the three shunts is identical for both modes 1 and 3 , while it is identical for the first $\left(Z_{1}\right)$ and third $\left(Z_{3}\right)$ absorbers for mode 2 . This is because the three absorbers are positioned symmetrically and thus perform almost equally well for the first three vibration modes, except for the second absorber $\left(Z_{2}\right)$ in mode 2 with vanishing influence.

The amplitudes of the frequency response functions (FRF) around vibration modes 1, 2 and 3 are now determined in order to verify the obtained shunt tuning in Table 3 . It is noted that the FRFs are only presented for the model with optimum parallel shunts, as the corresponding curves for the series shunts are very similar. The FRFs are obtained by applying the modal load $\left\{f_{r}\right\}=$ $[M]\left\{U_{r}\right\}$ to the structure for which the three parallel shunts are tuned. The magnitude of the FRFs in the point of maximum modal deflection $\left|u_{1}\right|,\left|u_{2}\right|$ and $\left|u_{3}\right|$ are in Figure $6(\mathrm{a}, \mathrm{c}, \mathrm{e})$ normalized by the corresponding static deflection $u_{0}$, while the corresponding voltage amplitudes $\left|V_{1}\right|,\left|V_{2}\right|$ and $\left|V_{3}\right|$ are shown in Figure $6(\mathrm{~b}, \mathrm{~d}, \mathrm{f})$. The term FRF will in the remainder of the paper refer to the magnitude of the frequency response function, and specifically for the normalized structural amplitudes it denotes therefore the dynamic amplification factor (DAF). In Figure 6(a,c,e) the horizontal dashed line indicates the dynamic amplification factor $\mathrm{DAF}=1 /\left(2 \zeta_{r}\right)$ at resonance $\omega=\omega_{r}$, with the damping ratios $\zeta_{r}$ determined from Table 1. It can be seen that flat plateaus in $\mathrm{DAF}=|\mathrm{FRF}|$ agrees well with the estimated damping level. Furthermore, it can be seen from Figure $6(\mathrm{~b}, \mathrm{~d}, \mathrm{f})$ that completely flat plateaus are obtained for the voltage response amplitudes $\left|V_{i}\right|$ normalized by the corresponding modal charges $\left|Q_{r}\right|_{i}$ around the resonance. For mode 1 the flat plateaus are at $\left|V_{2}\right|\left|Q_{1}\right|_{2} \simeq 0.5$ for shunt $Z_{2}$ and $\left|V_{1}\right|\left|Q_{1}\right|_{1}=\left|V_{3}\right|\left|Q_{1}\right|_{3} \simeq 0.25$ for shunt $Z_{1}$ and $Z_{3}$, which corresponds to the fractions $\left(\left(\hat{V}_{r}\right)_{i}\left(Q_{r}\right)_{i}\right) /\left(\left\{\hat{V}_{r}\right\}^{t}\left\{Q_{r}\right\}\right)$ appearing in the characteristic equation (27). This fraction also determines the ratio between the effective and modified effective 

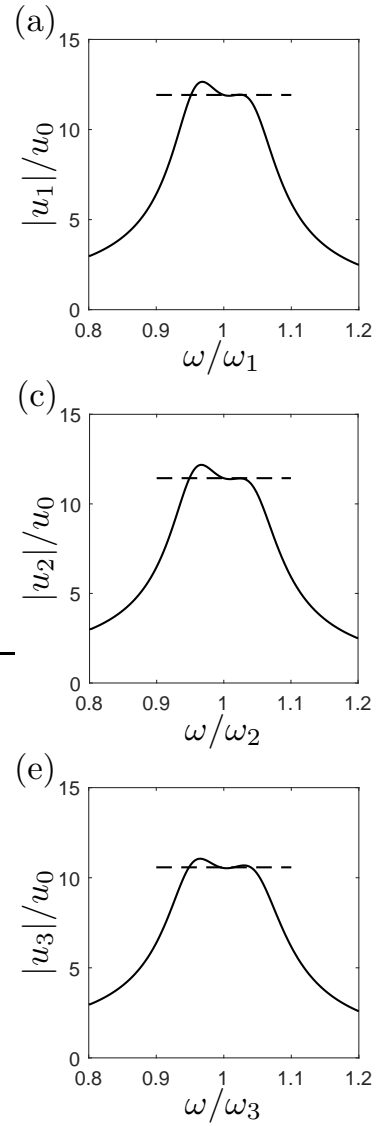
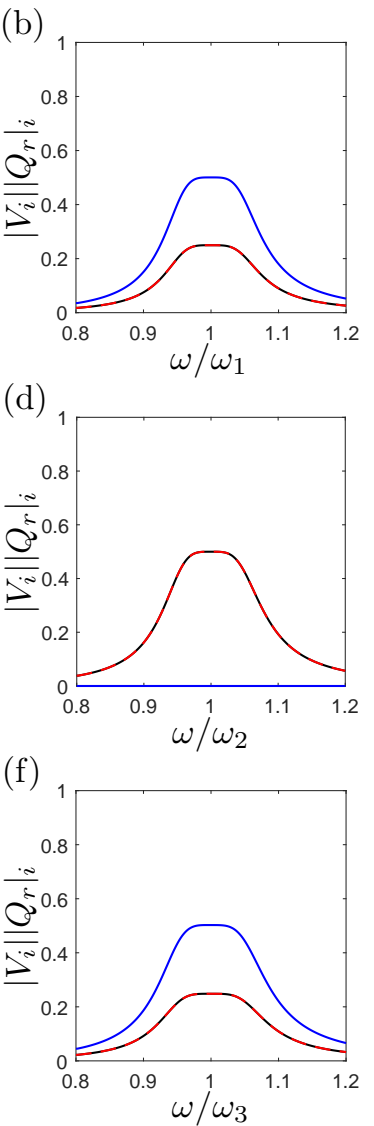

FigURE 6. FRF for the displacements (at the position of the maximum modal deflection) and voltages around mode $1(\mathrm{a}, \mathrm{b}), 2(\mathrm{c}, \mathrm{d})$ and $3(\mathrm{e}, \mathrm{f})$ of the simply supported beam with three parallel shunted piezoelectric patch pairs (absorbers). In $(\mathrm{a}, \mathrm{c}, \mathrm{e})$ the horizontal dashed lines indicate the DAFs, and in $(\mathrm{b}, \mathrm{d}, \mathrm{f})$ the black, blue and red lines indicates the voltage responses $V_{1}, V_{2}$ and $V_{3}$ over each absorber.

EMCCs in (11) and thus quantifies the amount of damping provided by each piezoelectric shunt. For mode 2 in Figure $6(\mathrm{~d})$ it is seen that the first $\left(Z_{1}\right)$ and third $\left(Z_{3}\right)$ piezoelectric absorbers each provide half of the total damping, while the second absorber $\left(Z_{2}\right)$ instead contributes the most to the damping of mode 3 .

5.2. Double mode damping. Simultaneous damping of the resonant vibration modes 1 and 2 in Figure $5(\mathrm{a}, \mathrm{b})$ is now considered. The second piezoelectric absorber $\left(Z_{2}\right)$ is tuned to vibration mode 1 , for which it is optimally positioned, while the first $\left(Z_{1}\right)$ and third $\left(Z_{3}\right)$ absorbers are tuned to vibration mode 2 . In the following the calibration strategies for the single-mode in the section Single-mode calibration, the modified single-mode in Modified single-mode calibration and the multi-mode in Multi-mode calibration with non-resonant absorbers are applied.

The single-mode tuning parameters are provided in Table 3 , with the second absorber $\left(Z_{2}\right)$ tuned to mode 1 and the first $\left(Z_{1}\right)$ and third $\left(Z_{3}\right)$ absorbers tuned to mode 2. According to (36) the single-mode calibration causes a decrease in the obtained damping ratio and ultimately a higher maximum DAF at resonance. It may therefore be beneficial to use the resistance tuning 
TABLE 4. Modal charge and voltage and optimum modified single and multimode parallel and series shunt tuning for the second piezoelectric absorber tuned to mode 1 and the first and third absorbers tuned to mode 2 .

\begin{tabular}{|c|c|c|c|c|c|c|c|}
\hline Shunt & $\left|Q_{r}\right|_{i}$ & $\left.m\left(\frac{C s}{r a d}\right)^{2}\right]$ & $\left|\hat{V}_{r}\right|_{i}[\mathrm{k} V]$ & $L_{i}^{p}[\mathrm{H}]$ & $R_{i}^{p}[\mathrm{k} \Omega]$ & $L_{i}^{s}[\mathrm{H}]$ & $R_{i}^{s}[\mathrm{k} \Omega]$ \\
\hline \multirow{2}{*}{ 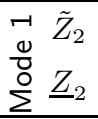 } & & 3.781 & 46.64 & 493.0 & 656.8 & 482.8 & 9.130 \\
\hline & & 3.781 & 46.32 & 489.6 & 654.5 & 479.4 & 9.035 \\
\hline \multirow{4}{*}{$\begin{array}{ll}\sim & \tilde{Z}_{1} \\
\sim & \tilde{Z}_{3} \\
\frac{0}{\circ} & \\
\Sigma & \underline{Z}_{1} \\
\underline{Z}_{3}\end{array}$} & & 16.07 & 198.41 & 29.62 & 109.3 & 28.73 & 3.269 \\
\hline & & 16.07 & 198.41 & 29.62 & 109.3 & 28.73 & 3.269 \\
\hline & & 16.07 & 198.41 & 29.62 & 109.3 & 28.73 & 3.269 \\
\hline & & 16.07 & 198.41 & 29.62 & 109.3 & 28.73 & 3.269 \\
\hline
\end{tabular}

from the modified effective EMCC (37), which gives

$$
\begin{array}{r}
\tilde{\kappa}_{1}^{2}=\frac{\left(Q_{1}\right)_{2}\left(\hat{V}_{1}\right)_{2}}{\omega_{1}^{2}}=0.70 \%, \\
\tilde{\kappa}_{2}^{2}=\frac{\left(Q_{2}\right)_{1}\left(\hat{V}_{2}\right)_{1}+\left(Q_{2}\right)_{3}\left(\hat{V}_{2}\right)_{3}}{\omega_{2}^{2}}=1.53 \%
\end{array}
$$

for the two modes. The optimum tuning of shunts $\tilde{Z}_{1}, \tilde{Z}_{2}$ and $\tilde{Z}_{3}$ is provided in Table 4 and determined by using the modified single-mode calibration from the second row of Table 1 .

Finally, the multi-mode calibration method presented in the third row of Table 1 is considered. This method requires the evaluation of two eigenvalue problems for each target mode, in order to obtain more representative effective EMCCs and modal charges and voltages. The two eigenvalue problems for the first target mode $(r=1)$ is obtained by grounding all absorber electrodes $V_{1}=$ $V_{2}=V_{3}=0$ in the SC case, while subsequently removing the constraint on the second absorber electrode $V_{1}=Q_{2}=V_{3}=0$ in the OC case. For the second target mode $(r=2)$ the SC eigenvalue problem is defined by keeping the second absorber in the $\mathrm{OC}$ condition, while the first and third absorbers are shorted: $V_{1}=Q_{2}=V_{3}=0$, while the OC eigenvalue problem has all absorbers in their OC condition: $Q_{1}=Q_{2}=Q_{3}=0$. Hereby, the corresponding two effective EMCCs are found as

$$
\underline{\kappa}_{1}^{2}=0.70 \%, \quad \underline{\kappa}_{2}^{2}=1.53 \%
$$

corresponding exactly to those in (44) obtained by the modified single-mode calibration. The new modal charges and voltages are provided in Table 4 together with the optimum parallel and series shunt tuning determined by the formulas in the third row of Table 1.

It can be seen from Table 4 that the modified single-mode and the multi-mode shunt tuning of $Z_{1}$ and $Z_{3}$ to mode 2 are identical and equal to the single-mode calibration in Table 3. This is because the second piezoelectric absorber $\left(Z_{2}\right)$ does not influence mode 2 . The single-mode calibration is therefore valid in this case without any reduction in attainable damping. The optimum tuning of the second shunt $Z_{2}$ to mode 1 is however seen to differ from the single-mode calibration in Table 3 , because of the influence from the first $\left(Z_{1}\right)$ and third $\left(Z_{3}\right)$ piezoelectric absorbers on the effective EMCC. The inductance tuning for the modified single-mode and the multi-mode calibrations in Table 4 are almost equal to those for the single-mode calibration in Table 3, since the effective EMCC only plays a minor role in the corresponding tuning formulas and the new modal charge and voltage only alter the inductance tuning of $\underline{Z}_{2}$ slightly. However, the resistance tuning for the modified single-mode and multi-mode calibrations in Table 4 are significantly different for mode 1 compared to those for the single-mode calibration in Table 3 , due to the modified effective EMCCs in (44) and (45).

The effect of using the three different shunt tuning methods is now analyzed by inspecting the FRFs in Figure 7 to a load with a spatial distribution equal to either the first or second resonant vibration modes. It can be seen from the FRF around the first resonant frequency 

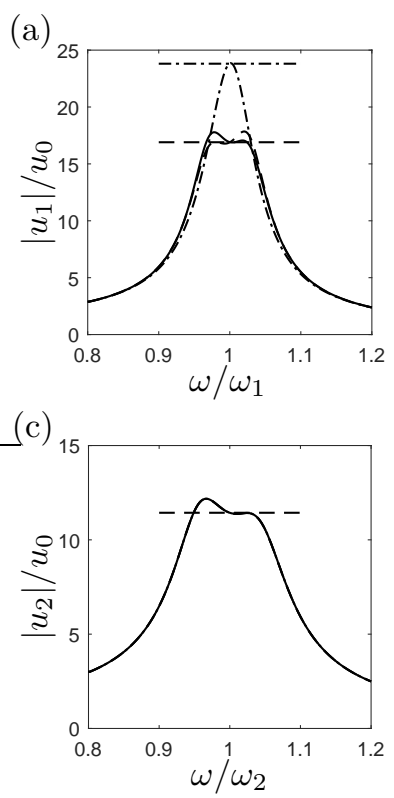

(b)

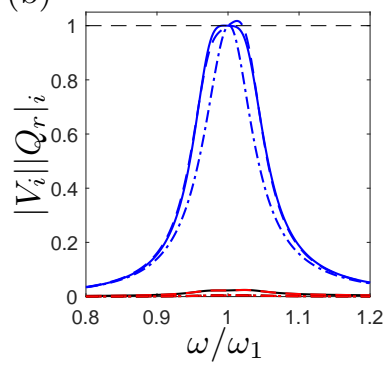

(d)

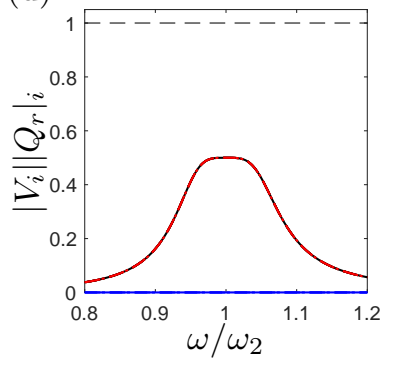

FiguRe 7. FRF to modal loads in mode $1(\mathrm{a}, \mathrm{b})$ and $2(\mathrm{c}, \mathrm{d})$, for parallel multimode (-), modified single-mode (--) and single-mode (--.) tuning of the piezoelectric absorbers. In (a,c) the horizontal dashed and dashed-dotted lines indicate, the DAFs based on the damping ratios (43) and (36), while the black, blue and red curves in (b,d) indicate the voltage responses $V_{1}, V_{2}$ and $V_{3}$, respectively.

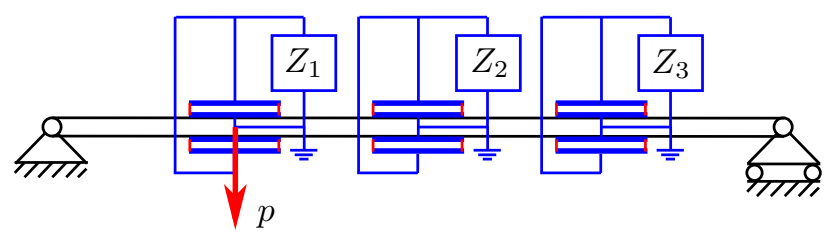

Figure 8. Position of dynamic point load $p$ on simply supported beam with three piezoelectric absorbers.

in Figure 7(a) that the single-mode calibration (dashed-dotted curve) causes a non flat plateau with a maximum DAF accurately determined from the reduced damping ratio in (36) (horizontal dashed-dotted line). When instead using the modified single-mode calibration (dashed curve) and the multi-mode calibration (solid curve), flat plateaus are observed for the corresponding FRFs in Figure 7(a). For the shunt voltage in Figure 7(b) the multi-mode calibration (solid curve) gives a slightly more flat plateau in the FRF compared to the modified single-mode calibration (dashed curve). It can be seen from the FRF around the second resonant frequency in Figure 7(c) that the three-shunt calibrations give identical responses, as $Z_{2}$ has no influence on mode 2 . For the mode 2 shunt voltage in Figure $7(\mathrm{~d})$ it is furthermore seen that the first $\left(Z_{1}\right)$ and third $\left(Z_{3}\right)$ absorbers contribute equally to the mitigation of mode 2 in Figure 6(d).

The simultaneous shunt tuning and damping of modes 1 and 2 of the simply supported beam is now analyzed by considering a dynamic point load located at the center point in Figure 8, of the first piezoelectric absorber where it spatially excites both vibration modes 1 and 2 . The FRF to the point load is seen in Figure 9 in a frequency spectrum capturing the first two resonances of the simply supported beam. As observed for the response to the modal loads in Figure 7, it can 


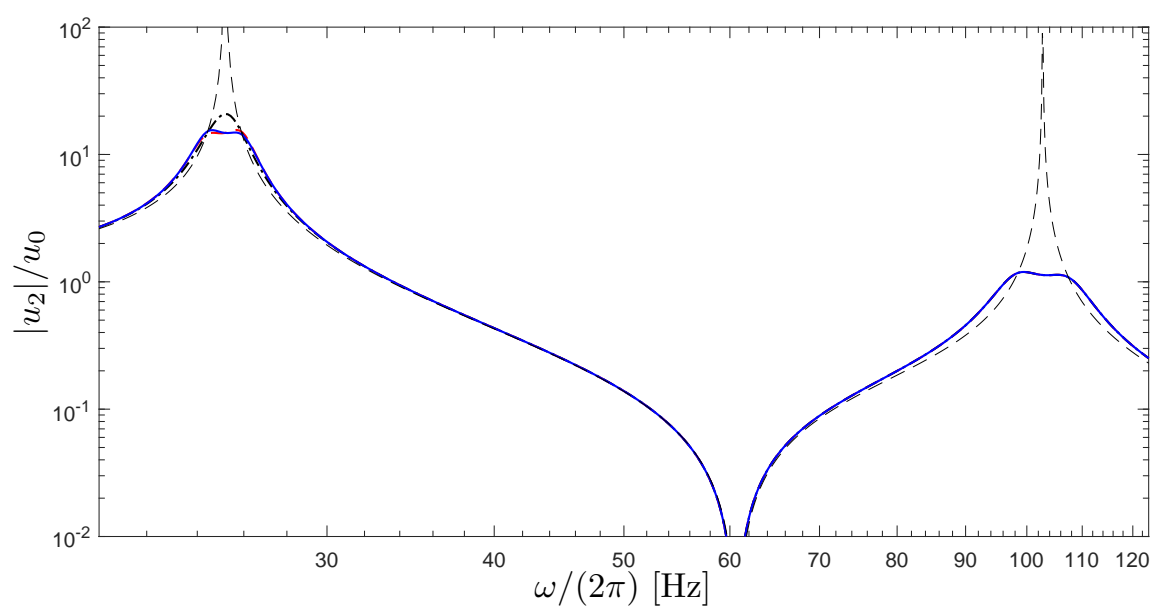

FiguRE 9. FRF for simply supported beam with point load in Figure 8, for parallel multi-mode tuning (-), modified single-mode tuning (-- -), single-mode tuning (-- ), and for the SC structure (-- -).

be seen in Figure 9 that optimum vibration suppression is obtained around mode 2 for all three tuning methods, while optimum suppression of vibration mode 1 requires the use of the modified single-mode tuning or the full multi-mode calibration. In all cases the three piezoelectric absorbers suppresses both resonant peaks significantly.

5.3. Triple mode damping. Simultaneous damping of the first three resonant vibration modes of the simply supported beam in Figure 4 is now considered and realized by tuning each of the three piezoelectric shunts to their individual vibration mode. The first piezoelectric absorber $\left(Z_{1}\right)$ is tuned to mode 2 , the second $\left(Z_{2}\right)$ to target mode 3 and the third $\left(Z_{3}\right)$ will be tuned to mode 1. Again, all three tuning methods are considered and compared. The single-mode calibration follows from Table 3, while the modified single-mode calibration follows from determination of the modified effective EMCC in (37), obtained for the three vibration modes as

$$
\begin{aligned}
& \tilde{\kappa}_{1}^{2}=\frac{\left(Q_{1}\right)_{3}\left(\hat{V}_{1}\right)_{3}}{\omega_{1}^{2}}=0.35 \%, \\
& \tilde{\kappa}_{2}^{2}=\frac{\left(Q_{2}\right)_{1}\left(\hat{V}_{2}\right)_{1}}{\omega_{2}^{2}}=0.76 \%, \\
& \tilde{\kappa}_{3}^{2}=\frac{\left(Q_{3}\right)_{2}\left(\hat{V}_{3}\right)_{2}}{\omega_{3}^{2}}=0.90 \%
\end{aligned}
$$

The corresponding optimum tuning of the parallel and series shunt $\tilde{Z}_{i}$ are provided in Table 5 . The multi-mode calibration requires the evaluation of individual eigenvalue problems in order to determine three new SC and $\mathrm{OC}$ resonance frequencies and the corresponding modal charges and voltages. The SC and OC eigenvalue problems (40) and (41) are respectively established for target mode $r=1$ by $V_{1}=V_{2}=V_{3}=0$ and $V_{1}=V_{2}=Q_{3}=0$, for target mode $r=2$ by $V_{1}=V_{2}=Q_{3}=0$ and $Q_{1}=V_{2}=Q_{3}=0$ and for target mode $r=3$ by $Q_{1}=V_{2}=Q_{3}=0$ and $Q_{1}=Q_{2}=Q_{3}=0$. The corresponding effective EMCCs are then found as

$$
\underline{\kappa}_{1}^{2}=0.35 \%, \quad \underline{\kappa}_{2}^{2}=0.76 \%, \quad \underline{\kappa}_{3}^{2}=0.89 \%
$$

while three new modal charge and voltage amplitudes are provided in Table 5. Finally, the optimum multi-mode parallel and series shunt tuning are given in Table 5.

It can be seen from Table 5 that the parallel shunt inductance for the modified single-mode calibration is identical to the single-mode calibration in Table 3 because the corresponding tuning formula (30) is independent of the effective EMCC. For the multi-mode calibration the inductance 
TABLE 5. Modal charge and voltage and optimum single and multi-mode parallel and series shunt tuning for the three piezoelectric absorbers dedicated to damp resonant vibration mode 2,3 and 1 , respectively.

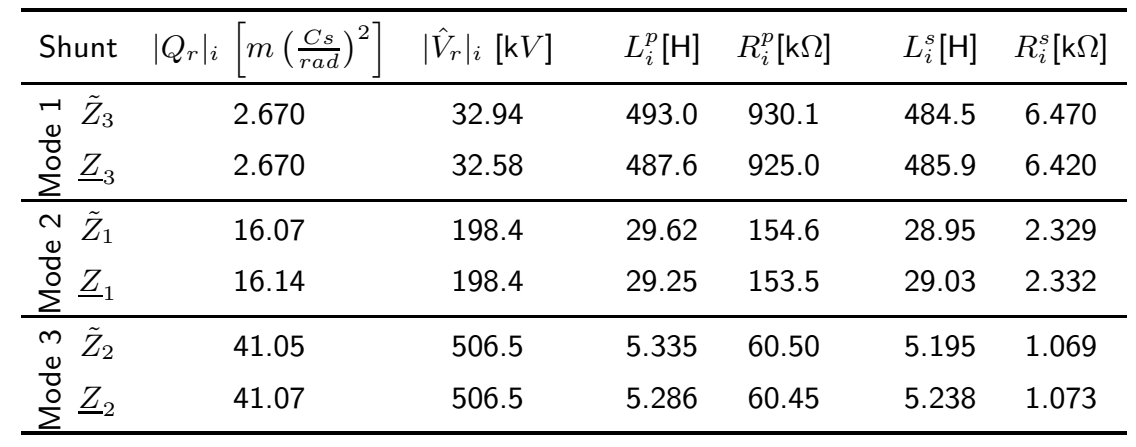

is slightly altered due to the new modal charges and voltages. As also found for the double mode damping, the optimum resistances in Table 5 are significantly different from the singlemode calibration values in Table 3, mainly due to the new effective EMCCs in (46) and (47). The three tuning methods are analyzed by inspecting the corresponding FRFs produced by applying modal loads for the respective three resonant vibration modes, shown for the parallel shunts in Figure 10.

It can be seen from Figure 10(a,c,e) that the maximum DAF at resonance is accurately determined from the reduced damping ratio (dashed line) in (36), when the single-mode calibration in Table 3 is used. Both the modified single-mode and multi-mode calibration give almost flat plateaus in the FRF based on the damping ratio in (43). A slightly higher amplitude may be observed for the modified single-mode calibration, due to the inaccuracy of the modal charges and voltages. For the shunt voltage in Figure 10 it is similarly observed that the multi-mode calibration is closer to the ideally balanced calibration with completely flat plateaus.

Finally, the performance of the shunt tuning provided in Table 3 and 5 are again analyzed by the FRF in Figure 11 for the harmonic point load in Figure 8. It can be seen from Figure 11 that the multi-mode calibration (blue solid curve) leads to the most flat plateaus around the resonant frequencies. The modified single-mode calibration (red dashed curve) provides almost the same vibration suppression around the three resonant frequencies and may thus be beneficial, as it avoids the evaluation of additional eigenvalue problems. The single-mode calibration (black dashed-dotted curve) is seen to cause non-flat plateaus around the resonant frequencies and a larger DAF, which is however still significantly reduced compared to the DAF for the SC structure (black dashed curve).

\section{Conclusion}

A resonant piezoelectric shunt calibration principle accounting for the influence of non-resonant vibration modes is proposed for multiple shunted piezoelectric domains (absorbers) attached to a flexible structure. The method requires the evaluation of two eigenvalue problems associated with the SC and $\mathrm{OC}$ conditions of the piezoelectric domains, from which the frequencies, modal charge and modal voltage are determined, constituting the required shunt tuning parameters. When only a subset of the piezoelectric shunts are tuned with respect to the target vibration mode the tuning becomes non-optimal and the attainable damping decreases. In this case an enhanced shunt tuning may be obtained by the determination of a modified effective EMCC, which only considers the piezoelectric absorbers tuned with respect to the target vibration mode. Finally, the tuning method is generalized to multi-mode vibration suppression, for which the corresponding optimum shunt tuning requires the evaluation of additional eigenvalue problems to account for the influence from piezoelectric absorbers tuned to modes below and above the target mode. 

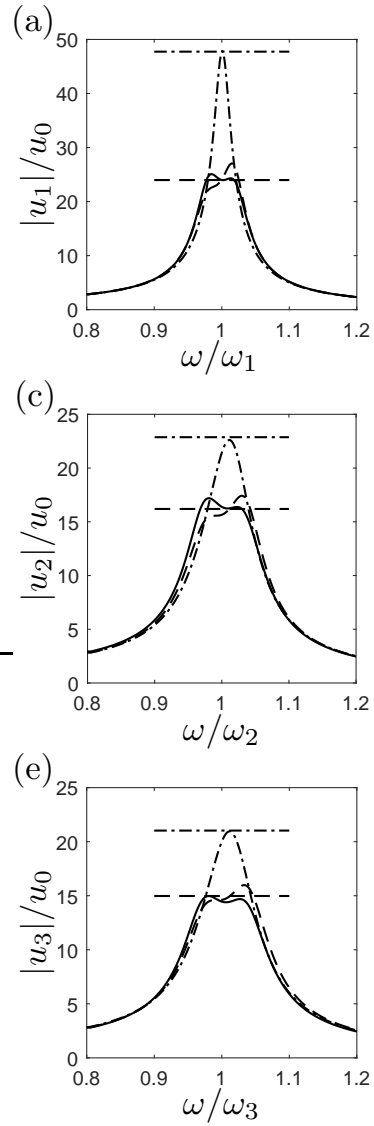

(b)

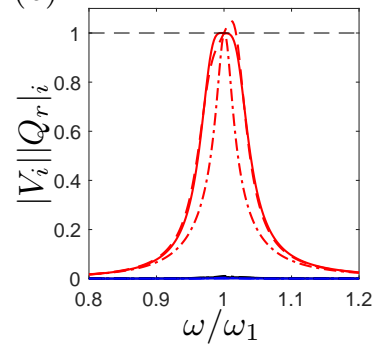

(d)

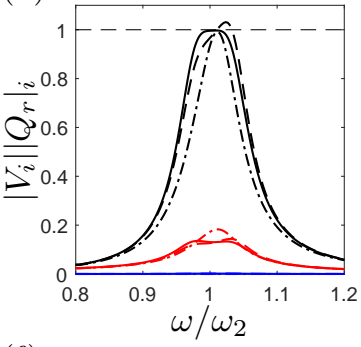

(f)

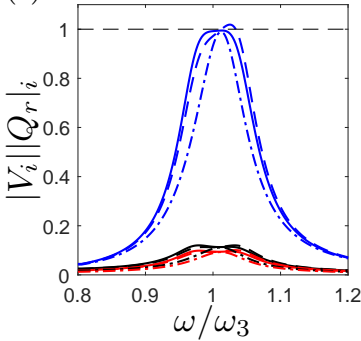

FiguRE 10. FRF to modal loads in mode $1(\mathrm{a}, \mathrm{b}), 2(\mathrm{c}, \mathrm{d})$ and $3(\mathrm{e}, \mathrm{f})$, for parallel multi-mode (-), modified single-mode (-- -) and single-mode (---) calibrations. In (a,c,e) the horizontal dashed and dashed-dotted lines indicate the DAFs based on the damping ratios (43) and (36), and in (b,d,f) the black, blue and red curves indicate the voltage responses $V_{1}, V_{2}$ and $V_{3}$, respectively.

The three shunt calibration methods are tested on a simple numerical example concerning a simply supported beam with three pairs of shunted piezoceramic patches (absorbers). First, it is demonstrated that the tuning of the three piezoelectric absorbers to the same resonant vibration mode provides significant vibration suppression and reduces the maximum DAF to the level predicted by an estimated modal damping ratio. It is found that the tuning parameters for the three shunts are indistinguishable, except for the tuning of the second absorber to the second vibration mode, since this absorber is exactly located at the nodal point of mode 2 .

Secondly, simultaneous damping of vibration modes 1 and 2 is considered. It is demonstrated that the single-mode calibration of the second piezoelectric absorber causes a non-optimal response around mode 1 , while a flat plateau is obtained around mode 2, damped by the piezoelectric absorbers 1 and 3 . For the first vibration mode the influence from absorber 1 and 3 on the effective EMCC causes the non-optimum damping, while the single-mode calibration remains valid for the second vibration mode, as the second absorber is located in nodal point. The optimum tuning to the first resonant mode may be enhanced by using the modified effective EMCC or the multimode calibration, where improved accuracy of the latter comes at the cost of evaluating additional eigenvalue problems. 


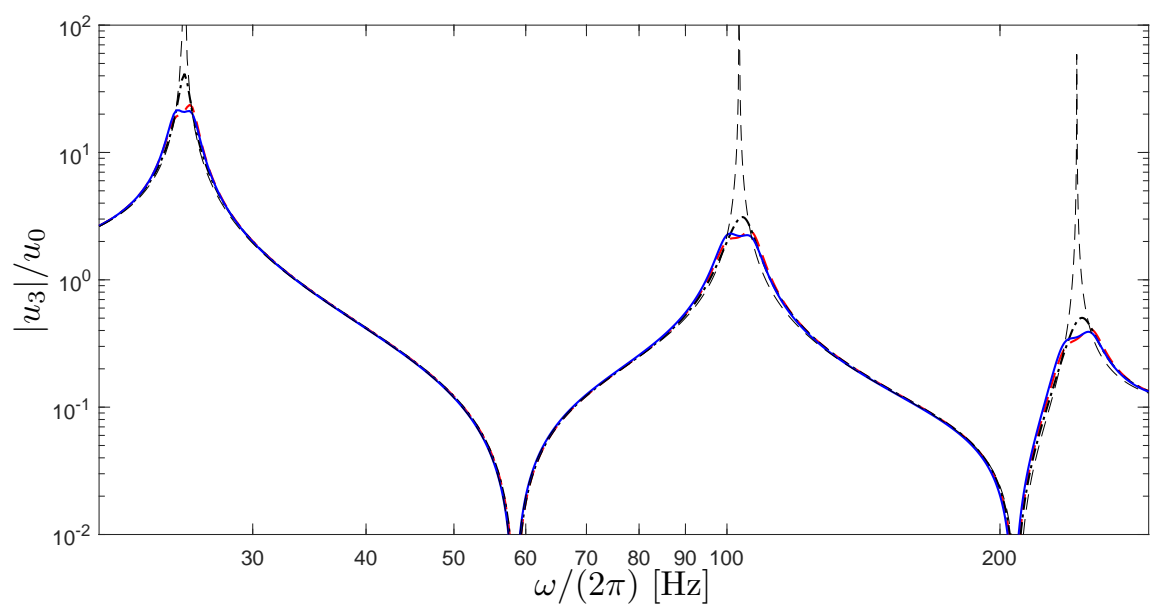

FiguRE 11. FRF for simply supported beam with point load in Figure 8, for parallel multi-mode (- $)$, modified single-mode (-- -) and single-mode (-.-.) calibrations, and for the SC structure (- - ).

Finally, simultaneous damping of the first three resonant vibration modes is considered. In this case the single-mode calibration leads to non-optimum shunt damping for all three vibration modes with a maximum DAF accurately estimated from a reduced modal damping ratio. However, when using the modified single-mode or the multi-mode calibrations, flat plateaus around the resonant frequencies are obtained in the corresponding FRFs. The results of the numerical example therefore indicate that it may be beneficial to use the modified single-mode calibration method, by which an optimum tuning and vibration suppression is obtained without the evaluation of the additional eigenvalue problems required in the full multi-mode calibration.

\section{FUNDING}

This research has been supported by the Danish Council for Independent Research via the project 'Resonant Piezoelectric Shunt Damping of Structures'.

\section{REFERENCES}

[1] Anderson E H and Hagood N W (1994) Simultaneous piezoelectric sensing/actuation: Analysis and application to controlled structures. J. Sound Vib. 174:617-39.

[2] Berardengo M, Thomas O, Giraud-Audine C and Manzoni S (2016) Improved resistive shunt by means of negative capacitance: new circuit, performances and multi-mode control. Smart Mater. and Struct. 25:075033(23pp).

[3] Berardengo M, Manzoni S, Thomas O, and Vanali M (2018) Piezoelectric resonant shunt enhancement by negative capacitances: Optimisation, performance and resonance cancellation. J. Intell. Mater. Syst. Struct. 29(12):2581-2606.

[4] Caruso G (2001) A critical analysis of electric shunt circuits employed in piezoelectric passive vibration damping. Smart Mater. Struct. 10:1059-68.

[5] Ducarne J,Thomas O and Deü J F (2010) Structural Vibration Reduction by Switch Shunting of Piezoelectric Elements: Modeling and Optimization. J. Intell. Mater. Syst. Struct. 21:797-816.

[6] eFunda Portal Lead Zirconate Titanate (PZT-5H). http://www.efunda.com/materials/piezo/ material_data/matdata_output.cfm ?Material_ID=PZT-5H (accessed 1 July 2019).

[7] Fleming A J, Behrens S and Moheimani S O R (2000) Synthetic impedance for implementation of piezoelectric shunt-damping circuits. Electronics Letters, 36:1525-6. 
[8] Forward R L (1979) Electronic damping of vibrations in optical structures. Appl. Optics. 18:690-7.

[9] Gardonio P and Casagrande D (2017) Shunted piezoelectric patch vibration absorber on two-dimensional thin structure: tuning considerations. J. Sound Vib. 395:26-47.

[10] Hagood N W and von Flotow A (1991) Damping of structural vibrations with piezoelectric materials and passive electrical networks. J. Sound Vib. 146:243-68.

[11] Hollkamp J J (1994) Multimodal passive vibration suppression with piezoelectric materials and resonant shunts. J. Intell. Mater. Syst. Struct. 5:49-57.

[12] Høgsberg J and Krenk S (2012) Balanced calibration of resonant shunt circuits for piezoelectric vibration control. J. Intell. Mater. Syst. Struct. 23:1937-48.

[13] Høgsberg J and Krenk S (2017) Calibration of piezoelectric RL shunts with explicit residual mode correction. J. Sound Vib. 386:65-81.

[14] IEEE Inc. (1988) Standards on piezoelectricity ANS/IEEE Std 176-1987 USA.

[15] Krenk S and Høgsberg J (2016) Tuned resonant mass or inerter-based absorbers: Unified calibration with quasi-dynamic flexibility and inertia correction. Proc. R. Soc. A. 472:20150718(23pp).

[16] Krenk S and Høgsberg J (2013) Equal modal damping design for a family of resonant vibration control formats. J. Vib. Cont. 19:1294-315.

[17] Larbi W and Deü J-F (2019) Reduced order finite element formulations for vibration reduction using piezoelectric shunt damping. Appl. Acoust. 147:111-20.

[18] Lossouarn B, Aucejo M, Deü J F and Multon B (2017) Design of inductors with high inductance values for resonant piezoelectric damping. Sens. Act. A: Phys. 259:68-76.

[19] Lossouarn B, Aucejo M, Deü J-F and Cunefare K A (2018) Design of a passive electrical analogue for piezoelectric damping of a plate. J. Intell. Mater. Syst. Struct. 29(7):1301-1314.

[20] de Marneffe B and Preumont A (2008) Vibration damping with negative capacitance shunts: theory and experiment. Smart Mater. Struct. 17:035015.

[21] Park C and Inman D (1999) Uniform model for series R-L and parallel R-L shunt circuits and power consumption. Proc. SPIE 3668:797-804.

[22] Park C H and Inman D J (1999) A uniform model for series R-L and parallel R-L shunt circuits and power consumption. SPIE Proceedings 3668:797-804.

[23] Preumont A (2011) Vibration Control of Active Structures. An Introduction 3rd edition Springer Heidelberg.

[24] Soltani P, Kerschen G, Tondreau G and Deraemaeker A (2014) Piezoelectric vibration damping using resonant shunt circuits: an exact solution. Smart Mater. Struct. 23:125014(11pp).

[25] Thomas O, Ducarne J and Deü J F (2012) Performance of piezoelectric shunts for vibration reduction. Smart Mater. Struct. 21:015008(16pp).

[26] Toftekær J F, Benjeddou A, Høgsberg J and Krenk S (2018) Optimal piezoelectric RL shunt damping of plates with residual mode correction. J. Intell. Mater. Syst. Struct. 29:3346-70.

[27] Toftekær J F, Benjeddou A and Høgsberg J (2019) General numerical implementation of a new piezoelectric shunt tuning method based on the effective electromechanical coupling coefficient. Mech. Adv. Mater. Struc.

[28] Wu S Y (1996) Piezoelectric shunts with a parallel R-L circuit for structural damping and vibration control. In Proc. SPIE 2720:259-69.

[29] Wu S Y (1998) Method for multiple mode piezoelectric shunting with single PZT transducer for vibration control. J. Intell. Mater. Syst. Struct. 12:991-8.

[30] Yamada K, Matsuhisa H, Utsuno H and Sawada K (2010) Optimum tuning of series and parallel LR circuits for passive vibration suppression using piezoelectric elements. J. Sound Vib., 329:5036-57. 


\section{Appendix I}

The square brackets in (19) may be multiplied by $\mathrm{i} \omega[Z(\omega)]\left\{Q_{r}\right\}$, whereby the characteristic equation can be written as

$$
\begin{aligned}
& \left(1-\frac{\omega^{2}}{\omega_{r}^{2}}\right)\left(\mathrm{i} \omega \frac{\left\{\hat{V}_{r}\right\}^{t}\left[C_{r}\right][Z(\omega)]\left\{Q_{r}\right\}}{\left\{\hat{V}_{r}\right\}^{t}\left\{Q_{r}\right\}}+1\right) \\
& +\mathrm{i} \omega \frac{\left\{Q_{r}\right\}^{t}[Z(\omega)]\left\{Q_{r}\right\}}{\left\{Q_{r}\right\}^{t}\left[C_{r}\right]^{-1}\left\{Q_{r}\right\}} \kappa_{r}^{2}=0
\end{aligned}
$$

The series impedance (32) and effective capacitance (22) are now inserted in (48), whereby the characteristic equation can be written as

$$
\begin{gathered}
\left(1-\frac{\omega^{2}}{\omega_{r}^{2}}\right)\left(\omega^{2} \sum_{i=1}^{N_{p}} \frac{\left(\hat{V}_{r}\right)_{i}\left(Q_{r}\right)_{i}}{\left\{\hat{V}_{r}\right\}^{t}\left\{Q_{r}\right\}} L_{i}^{s} \frac{\left(Q_{r}\right)_{i}}{\left(\hat{V}_{r}\right)_{i}}\right. \\
\left.-\mathrm{i} \omega \sum_{i=1}^{N_{p}} \frac{\left(\hat{V}_{r}\right)_{i}\left(Q_{r}\right)_{i}}{\left\{\hat{V}_{r}\right\}^{t}\left\{Q_{r}\right\}} R_{i}^{s} \frac{\left(Q_{r}\right)_{i}}{\left(\hat{V}_{r}\right)_{i}}+1\right) \\
+\omega^{2} \sum_{i=1}^{N_{p}} \frac{\left(\hat{V}_{r}\right)_{i}\left(Q_{r}\right)_{i}}{\left\{\hat{V}_{r}\right\}^{t}\left\{Q_{r}\right\}} L_{i}^{s} \frac{\left(Q_{r}\right)_{i}}{\left(\hat{V}_{r}\right)_{i}} \kappa_{r}^{2} \\
-\mathrm{i} \omega \sum_{i=1}^{N_{p}} \frac{\left(\hat{V}_{r}\right)_{i}\left(Q_{r}\right)_{i}}{\left\{\hat{V}_{r}\right\}^{t}\left\{Q_{r}\right\}} R_{i}^{s} \frac{\left(Q_{r}\right)_{i}}{\left(\hat{V}_{r}\right)_{i}} \kappa_{r}^{2}=0
\end{gathered}
$$

It is convenient to divide (49) with $\sum_{i=1}^{N_{p}} \frac{\left(\hat{V}_{r}\right)_{i}\left(Q_{r}\right)_{i}}{\left\{\hat{V}_{r}\right\}^{t}\left\{Q_{r}\right\}} L_{i}^{s} \frac{\left(Q_{r}\right)_{i}}{\left(\hat{V}_{r}\right)_{i}} \omega_{r}^{2}$, which then can be written as

$$
\begin{aligned}
\left(1-\frac{\omega^{2}}{\omega_{r}^{2}}\right) & \left(\frac{\omega^{2}}{\omega_{r}^{2}}-\mathrm{i} \frac{\omega}{\omega_{r}} \frac{\sum_{i=1}^{N_{p}}\left(Q_{r}\right)_{i}^{2} R_{i}^{s}}{\sum_{i=1}^{N_{p}}\left(Q_{r}\right)_{i}^{2} L_{i}^{s} \omega_{r}}\right. \\
+ & \left.\sum_{i=1}^{N_{p}} \frac{\left\{\hat{V}_{r}\right\}^{t}\left\{Q_{r}\right\}}{\left(\hat{V}_{r}\right)_{i}\left(Q_{r}\right)_{i}} \frac{1}{L_{i}^{s}} \frac{\left(\hat{V}_{r}\right)_{i}}{\left(Q_{r}\right)_{i} \omega_{r}^{2}}\right) \\
+ & \frac{\omega^{2}}{\omega_{r}^{2}} \kappa_{r}^{2}-\mathrm{i} \frac{\omega}{\omega_{r}} \frac{\sum_{i=1}^{N_{p}}\left(Q_{r}\right)_{i}^{2} R_{i}^{s}}{\sum_{i=1}^{N_{p}}\left(Q_{r}\right)_{i}^{2} L_{i}^{s} \omega_{r}} \kappa_{r}^{2}=0
\end{aligned}
$$

By expanding the expressions in (50), it can be written as

$$
\begin{gathered}
\frac{\omega^{4}}{\omega_{r}^{4}}-\left(1+\kappa_{r}^{2}-\sum_{i=1}^{N_{p}} \frac{\left\{\hat{V}_{r}\right\}^{t}\left\{Q_{r}\right\}}{\left(\hat{V}_{r}\right)_{i}\left(Q_{r}\right)_{i}} \frac{1}{L_{i}^{s}} \frac{\left(\hat{V}_{r}\right)_{i}}{\left(Q_{r}\right)_{i} \omega_{r}^{2}}\right) \frac{\omega^{2}}{\omega_{r}^{2}} \\
-\sum_{i=1}^{N_{p}} \frac{\left\{\hat{V}_{r}\right\}^{t}\left\{Q_{r}\right\}}{\left(\hat{V}_{r}\right)_{i}\left(Q_{r}\right)_{i}} \frac{1}{L_{i}^{s}} \frac{\left(\hat{V}_{r}\right)_{i}}{\left(Q_{r}\right)_{i} \omega_{r}^{2}} \\
+\mathrm{i} \frac{\omega}{\omega_{r}} \frac{\sum_{i=1}^{N_{p}}\left(Q_{r}\right)_{i}^{2} R_{i}^{s}}{\sum_{i=1}^{N_{p}}\left(Q_{r}\right)_{i}^{2} L_{i}^{s} \omega_{r}}\left(1+\kappa_{r}^{2}-\frac{\omega^{2}}{\omega_{r}^{2}}\right)=0
\end{gathered}
$$

Finally, the characteristic equation (51) may be expressed in the generic form of (25) by division with $\left(1+\kappa_{r}^{2}\right)^{2}$ and introduction of the OC frequency from (7), which leads to (33). 